\title{
A new column collapse apparatus for the characterisation of the flowability of granular materials
}

\author{
J. Torres-Serra ${ }^{\mathrm{a}, \mathrm{b}, *}$, E. Romero ${ }^{\mathrm{b}}$, A. Rodríguez-Ferran ${ }^{\mathrm{b}}$ \\ ${ }^{a}$ Técnicas Mecánicas Ilerdenses, S.L., Polígono industrial Camí dels Frares, c. Alcarràs, \\ parc. 66, 25190 Lleida, Spain \\ ${ }^{b}$ Department of Civil and Environmental Engineering, Universitat Politècnica de \\ Catalunya, Campus Nord, c. Jordi Girona, 1-3, 08034 Barcelona, Spain
}

\begin{abstract}
The packaging industry is lacking a standard methodology to characterise the granular flow of a wide range of powders and grains in actual handling conditions. We present a new fully-instrumented granular column collapse apparatus for the experimental investigation of granular flow phenomena, by a quasi-twodimensional set-up with novel features including: a lifting gate activated by a parallelogram mechanism for material release; a reversible pneumatic circuit to impose fluidised and vacuum conditions to the initial granular column; a set of load cells to monitor the basal load distribution during flow propagation; a 3D laser line profile sensor to scan the free surface morphology of the samples at rest; and a high-speed video recording set to capture near-wall flow visualisations and relevant kinematic measures by particle image velocimetry. The selected results on dry flows of oat flakes, copper sulphate fertiliser, and talc powder samples show their distinctive flow dynamics, indicating the good flowability of fertiliser compared to the poor flowability of talc. This research has implications for the selection and design of bulk solids handling equipment, and the calibration and validation of mechanical and numerical models.
\end{abstract}

Keywords: granular column collapse, run-out, flow height, particle image velocimetry, flowability

\footnotetext{
${ }^{*}$ Corresponding author.

Email addresses: j.torres@tmipal.com (J. Torres-Serra), enrique.romero-morales@upc.edu (E. Romero), antonio.rodriguez-ferran@upc.edu (A. Rodríguez-Ferran)
} 


\section{Introduction}

A wide range of powders and grains are handled in the packaging industry using bulk solids handling equipment, among which bag filling systems are of our interest. The performance of bagging machinery and the packaging quality

5 rely on the first unit operation of dosing of the granular materials [1]. Dosing is carried out by gain-in-weight batching systems, consisting of a volumetric feeder that regulates the flow of bulk solids out of a supply hopper and into a weighing hopper [2]. Based on the applied material conveying techniques, a variety of feeder types exist [3]: free discharge by gravity feeders and rotary valves; positive displacement by belt, screw, and vibratory tray feeders; and pneumatic transport by fluidisation chambers. The operating efficiency of the feeders is critically affected by the flowability of the granular materials [4]. Nevertheless, it is still a common practice in the industry to base the feeder selection and design strategies solely on a qualitative assessment of flowability, the unreliability

15 of which entails financial and environmental costs throughout the life cycle of the equipment. Therefore, the lack of a standard protocol for characterising granu-lar flow phenomena remains a challenging issue in practical handling [5]. This motivates the development of experimental methodologies that can be equally applied to the diversity of powders and grains on the market. Then, robust classification strategies can be designed, relying on redundant material databases of the extracted parameters, combined with the engineering know-how and the mechanical behaviour modelling from experimental observations.

Flowability of granular materials involves complex mechanical behaviour issues, such as particle size segregation, affecting the homogeneity of free-flowing material batches [6], and jamming processes [7], which lead to the formation of stable arch and rathole structures [8, 9]. The constitutive behaviour of bulk solids is otherwise affected by environmental conditions such as the ambient temperature and the relative humidity, the latter affecting the amount of stored water in hygroscopic materials [10. In turn, the exposure to environmental con30 ditions alters flowability by controlling slip-stick and caking processes [11, 12]. The effect on flowability of the material properties of powders and grains produced by the food and agri-food, construction and mining, chemical and pharmaceutical, and recycling industries [13 17] has been studied using techniques 
at different scales. At the microscopic or grain scale, most work has focused on the investigation of the particle size, shape, and density; whereas at the macroscopic or bulk scale, attention has been paid to robust physical measurements including the compressibility, defined in terms of uniaxial compression stress, and shear testing in the quasi-static regime 18. Moreover, flowability for specific applications - for instance additive manufacturing or die filling - has been 40 researched respectively by avalanching and indentation [19, 20]. Other methodologies exist that provide conventional parameters, albeit with indirect physical interpretations, such as the discharge rates, compressibility in terms of tapping densities, or the angle of repose [21, 22].

Over the last two decades, granular column collapse experiments have re${ }_{45}$ ceived rising attention, aiming at understanding natural and industrial granular flows 23]. Fig. 1 shows the experimental set-up consisting of a reservoir in which the granular column is prepared and pre-conditioned. The reservoir wall is instantaneously removed, allowing the granular material to flow over the base, driven by gravity, until the final deposit configuration is attained at rest. We distinguish different types of granular column collapse experimental set-ups by their main features, as found in the literature:

- The geometry of the initial column has been considered to be either quasitwo-dimensional 25 31, assuming plane strain conditions inside a rectangular channel, or axi-symmetrical [23, 25, 29, 32, 36], which does not allow inspecting the internal structure of the mobilised mass during flow propagation. So-called semiaxisymmetric geometries have been explored to circumvent such drawbacks with consistent results compared to cylindrical set-ups [26]. For a quasi-two-dimensional set-up, we define the initial column aspect ratio as $a=h_{0} / l_{0}$, with $h_{0}$ the average initial granular column height after pre-conditioning, and $l_{0}$ the reservoir base length, see Fig. 1. The explored values of $a$ range typically between around 0.5 [25, 26, 31] and 10 30, 31.

- In the case of quasi-two-dimensional set-ups, the procedure for material release generally consists in the instantaneous removal of a vertically lifted gate, ensuring that the time to completely separate the gate from the granular material is shorter than the elapsed time for the onset of flow 32 . 


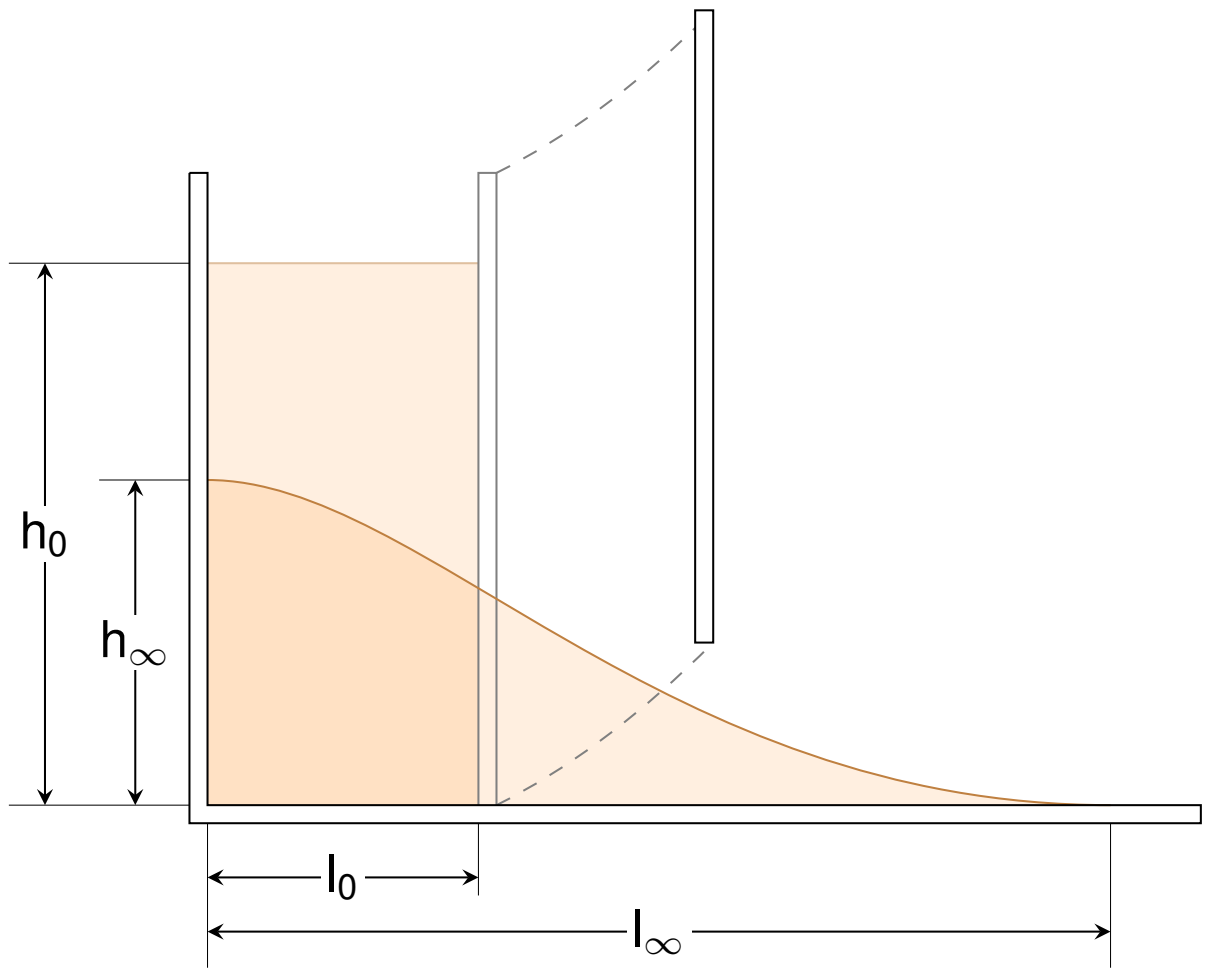

Fig. 1: Schematic of a quasi-two-dimensional granular column collapse experimental set-up, showing the initial granular column $\left(h_{0}, l_{0}\right)$ and final deposit $\left(h_{\infty}, l_{\infty}\right)$ configurations (adapted from [24]). 
Alternatively, some authors have resorted to swinging gates [27, 28, 37.

- The basal surface geometry and properties have been investigated. Beyond the usual horizontal surface configuration, the special case of a rotating horizontal table has been analysed in [34. A smooth basal surface has been adopted in most cases, whereas experiences with rough surfaces- e.g. covered with sandpaper of a roughness of the same order as the tested granular material - have shown little effect on the results [23. Furthermore, flows down rough inclined surfaces have been studied primarily in the field of earth sciences 38, 39 to understand phenomena such as landslides or debris flows. Other works have presented results from inclined plane observations with rough [40] and smooth [41, 42] basal surfaces. The maximum reported inclinations with respect to the horizontal range from 15 to $35^{\circ}$. Erodible beds, formed by layers of variable thickness of a granular material, have also shown negligible difference in the flow behaviour and deposit morphology on horizontal set-ups [32, whereas the flow dynamics and run-out have been confirmed to be controlled by erosion in the case of inclined channels or steps, as reviewed in 43 .

- The interstitial fluid surrounding the granular material, either gas or liquid, has been also taken into account. Dry granular flows have been mostly researched, without control of the packing state of the material. Air fluidisation of granular columns both previous to and during flow has been reported in 44, 45. The inclusion of a liquid phase, in the form of capillary water in the pendular state, has been examined in [46-48. Finally, fully saturated granular samples, immersed in distilled water or in various mixtures of water with soluble agents, have been tested in [49 [51]. The pore fluid pressures at the basal surface have been controlled in the cases of air fluidisation and fully immersed set-ups [4, 49.

We have developed a new fully-instrumented granular column collapse apparatus [52, 53, that provides for a direct observation of granular flow regimes generated in actual handling conditions [54, and suitable for a wide range of powders and grains with particle sizes from the scale of $\mu \mathrm{m}$ to $\mathrm{mm}$. The apparatus allows us to characterise the effect of their varying physical and mechanical 
properties at the grain and bulk scales on their flowability. Few researchers have addressed the question of the granular column collapse of fine-grained cohesive powders - for instance gypsum plaster [31 — and have largely focused on studying free-flowing non-cohesive coarser materials such as monodisperse spherical glass beads or quartz sand. We have equipped our experimental apparatus with complementary measurement techniques to visualise granular flow by looking at: the fluidisation and deaeration of the granular columns, the basal load distribution after collapse, the near-wall kinematics during flow propagation, and the free surface morphology of the final deposits. With the instrumentation redundancy, we seek to circumvent the eventual operational issues, as well as the problems associated with the material properties, such as the particle colour and granular texture affecting the feasibility of the image analysis of the flows.

This paper is organised as follows. First, we describe our experimental apparatus and the integrated instrumentation in section 2 Next, we present three representative granular materials, and we detail our testing protocol in section 3 . We illustrate the capabilities of the apparatus by following the testing protocol to analyse the chosen materials. We show and discuss a selection of the obtained results in section 4 . Last, we summarise the highlights of our work and draw our conclusions in the final section 5 ,

\section{Experimental set-up}

Fig. 2 shows views of our new fully-instrumented granular column collapse apparatus, consisting of a horizontal rectangular channel (1) of width $160 \mathrm{~mm}$ and length $2150 \mathrm{~mm}$, which comprises a smooth anodised aluminium plate at the base, and vertical glass walls of height varying between $350 \mathrm{~mm}$ to $150 \mathrm{~mm}$ for ease of operation. The prismatic configuration permits the thorough visualisation and instrumentation of the set-up, by exploiting the quasi-two-dimensional nature of the generated granular flows. This in turn facilitates the interpretation and modelling of the experimental results.

An automated lifting gate (2) is closed at a distance $l_{0}=150 \mathrm{~mm}$, forming a prismatic reservoir $33150 \times 150 \times 350 \mathrm{~mm}^{3}$ where the granular material column is prepared. The lightweight gate consists of a carbon fibre reinforced polymer sheet, held by a 3D-printed polymeric frame, and reinforced with an 

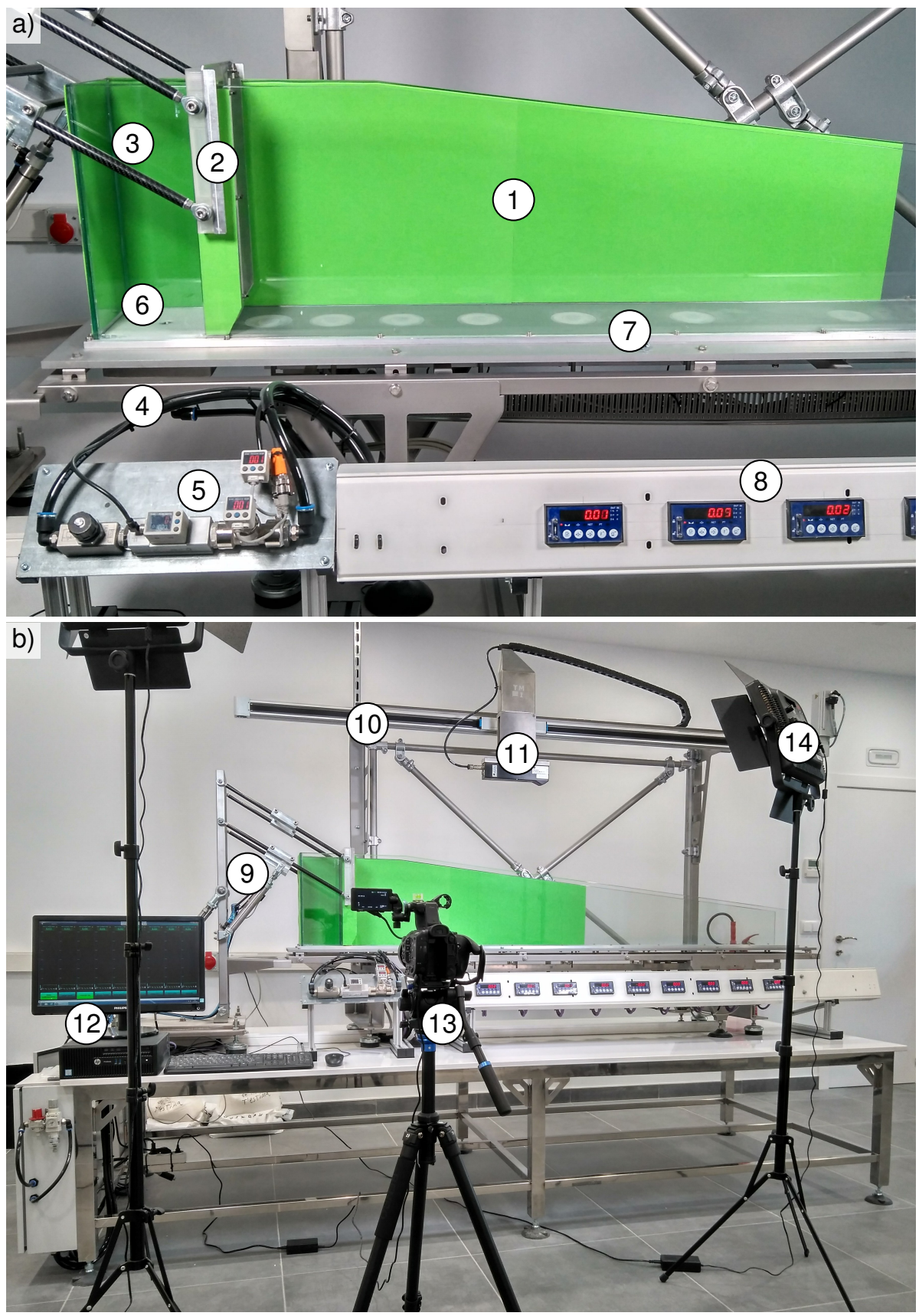

Fig. 2: New fully-instrumented apparatus with numbered parts. a) Close-up view of the channel: 1 . horizontal channel $\left(160 \times 2150 \mathrm{~mm}^{2}\right)$; 2. automated lifting gate; 3. reservoir $\left(150 \times 150 \times 350 \mathrm{~mm}^{3}\right) ; 4$. air chamber; 5 . air flow control panel; 6 . permeable plate; 7 . membranes and beam load cells; 8. signal filtering and transmission panel. b) General view of the experimental set-up: 9. automated parallelogram mechanism of the lifting gate; 10. linear guide held by a detachable structure; 11. 3D laser line profile sensor; 12 . PC for data acquisition and control; 13. high-speed video camera on tripod; 14. LED illumination. 
aluminium sheet. The gate opening is controlled by a parallelogram mechanism (9) automated by pneumatic cylinders, providing a uniformly accelerated circular motion of the gate, as illustrated in Fig. 3. A constant angular acceleration of $8 \mathrm{rad} \mathrm{s}^{-2}$ is achieved at an adjusted operating gauge pressure of the cylinders of $0.4 \mathrm{MPa}$, attaining a speed of $0.4-0.5 \mathrm{~m} \mathrm{~s}^{-1}$ after $0.1 \mathrm{~s}$ starting from repose. Our design addresses the main limitations of commonly used gate systems. First, the gate is instantaneously removed horizontally from its closed position, thus reducing the shear stresses on the granular column in contact with the gate during fast vertical lifting. Second, the parallelogram mechanism also reduces drag forces on the granular column caused by swinging gates in the direction of flow, to which materials with low particle density are more susceptible, while operating at a comparable velocity to the reported value of $0.4 \mathrm{~m} \mathrm{~s}^{-1} 37$.

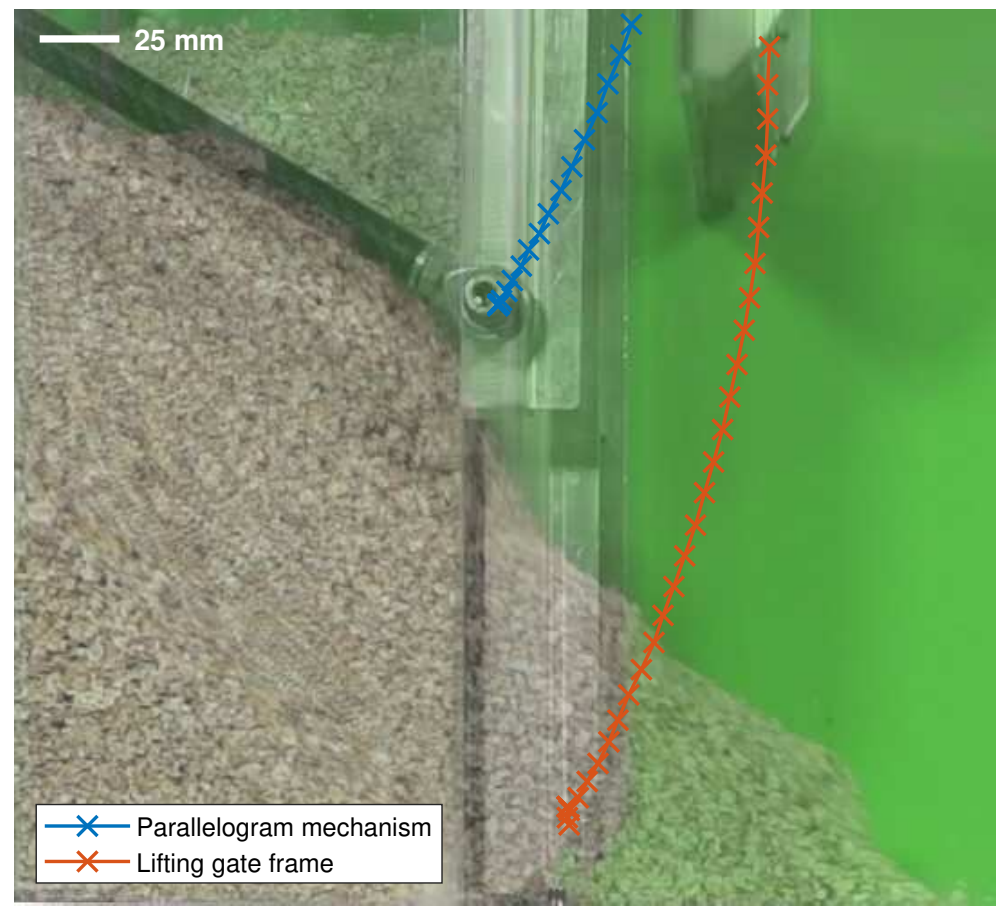

Fig. 3: Pathlines of two points on the lifting gate along the opening.

The initially poured random packing state of the granular column in the reservoir can be pre-conditioned either to a fluidised state of the material, obtained by injecting a positive air flow into the sample, or to a deaerated state of the material, generated under negative pressure conditions with the reversible 
pneumatic circuit depicted in Fig. 4. This circuit allows us to reproduce actual conditions in bulk solids handling operations: aeration in fluidisation chambers for bag filling [55], and deaeration to densify granular masses and prepare the package to be closed. A source of dried compressed air is connected through a solenoid valve to an air chamber (4) attached to the reservoir base. To create vacuum conditions inside the reservoir, the compressed air is redirected by the solenoid valve to a vacuum ejector to reverse the air flow out of the air chamber. A control panel (5) is installed between the solenoid valve and the air chamber, where the air flow is regulated and measured using a flowmeter (SMC Corporation of America, US) with a measuring range of $3 \times 10^{-4}$ to $3 \times 10^{-2} \mathrm{~m}^{3} \mathrm{~s}^{-1}$ and an accuracy of $\pm 0.05 \%$ FS. Two pressure transducers (SMC Corporation of America, US), with a measuring range of $\pm 100 \mathrm{kPa}$ and an accuracy of $\pm 0.05 \% \mathrm{FS}$, are also used to assess the air pressure inside the pneumatic circuit and, foremost, at the reservoir base, where a permeable polyester needle-punched felt plate $(6)$ is placed to prevent the escape of fine particles.

a)

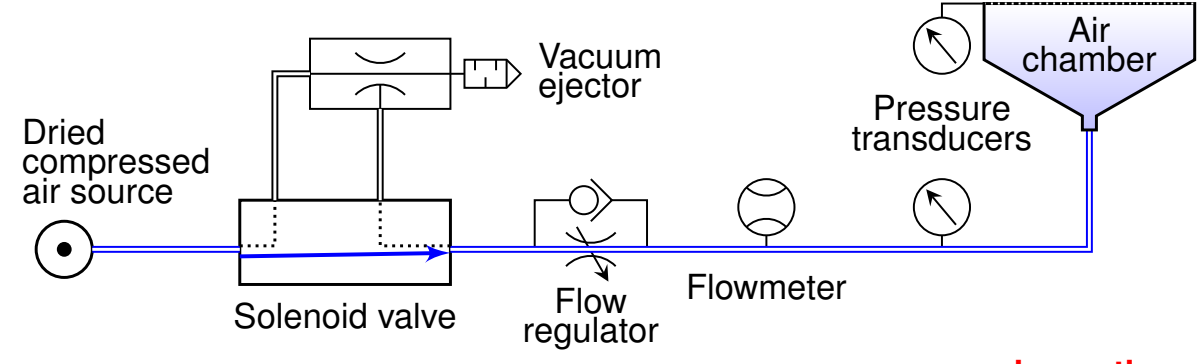

b)

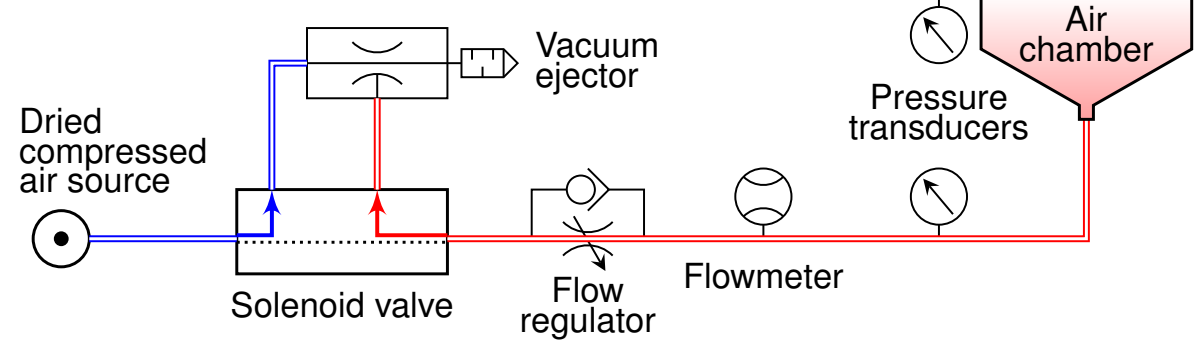

Fig. 4: Air flow diagram of the reversible pneumatic circuit with two operating modes: a) fluidisation; b) deaeration. The blue colour indicates positive air pressure and flow sense from the air source to the air chamber. The red colour indicates negative air pressure and flow sense from the air chamber to the vacuum ejector. 
Once the sample is prepared within the reservoir and its packing state preconditioned, the onset of flow is triggered by opening the gate synchronised in turn with a set of nine logarithmically-spaced beam load cells (UTILCELL, Spain) distributed along the channel base (7). The sensors are ordered by measuring range from 300 to $30 \mathrm{mN}$ with an accuracy of $\pm 0.02 \% \mathrm{FS}$, since the flow dynamics produce higher basal loading at channel locations closer to the reservoir. Embedded in the centre line of the channel base width are nine moulded silicone membranes, see Fig. 5a, that transmit the loads acting on the channel surface to the respective force transducers fixed to the channel structure, as shown in Fig. 5b. The membrane design is detailed in Fig. 5c consisting of a circular sheet of thickness $\theta=0.3 \mathrm{~mm}$, which deforms elastically under the action of the granular material deposited on it; a central block transmits the reaction force to the load cell on which it is supported. The load cells monitor flow propagation and load distribution of the granular material along the channel surface, through a set of high-speed transmitters (UTILCELL, Spain) installed on a signal filtering and transmission panel (8).

Aligned with the channel, a detachable structure holds a linear guide 10 covering the whole channel length with an effective stroke of $2200 \mathrm{~mm}$, and encasing a toothed belt with positioning control by a stepper motor with a repetition accuracy of $\pm 0.01 \%$ FS. Coupled to the slide (11) of the linear guide is a 3D laser line profile sensor (LMI Technologies Inc, Canada) positioned as depicted in Fig. 6. The profile sensor provides measurements of the top free surface morphology of the granular material at rest: first, of the granular column in the reservoir before testing; and second, of the final deposit along the channel after the flow stops. The analysis of the run-out is required to verify the quasi-two-dimensional hypothesis on the mechanical behaviour of the bulk material in the prismatic channel, as well as to observe shape parameters of the deposit such as the angle of repose [24, 29]. We consider the reference frame shown in Fig. 6, with the origin in the midpoint of the inner bottom left edge of the reservoir. Therefore, the spatial resolution of the profile sensor lies between $19-60 \mu \mathrm{m}$, with a range of $400 \mathrm{~mm}$ in the $z$-axis direction, and a field of view between 158 to $365 \mathrm{~mm}$ in the $y$-axis direction. We use a desktop computer 

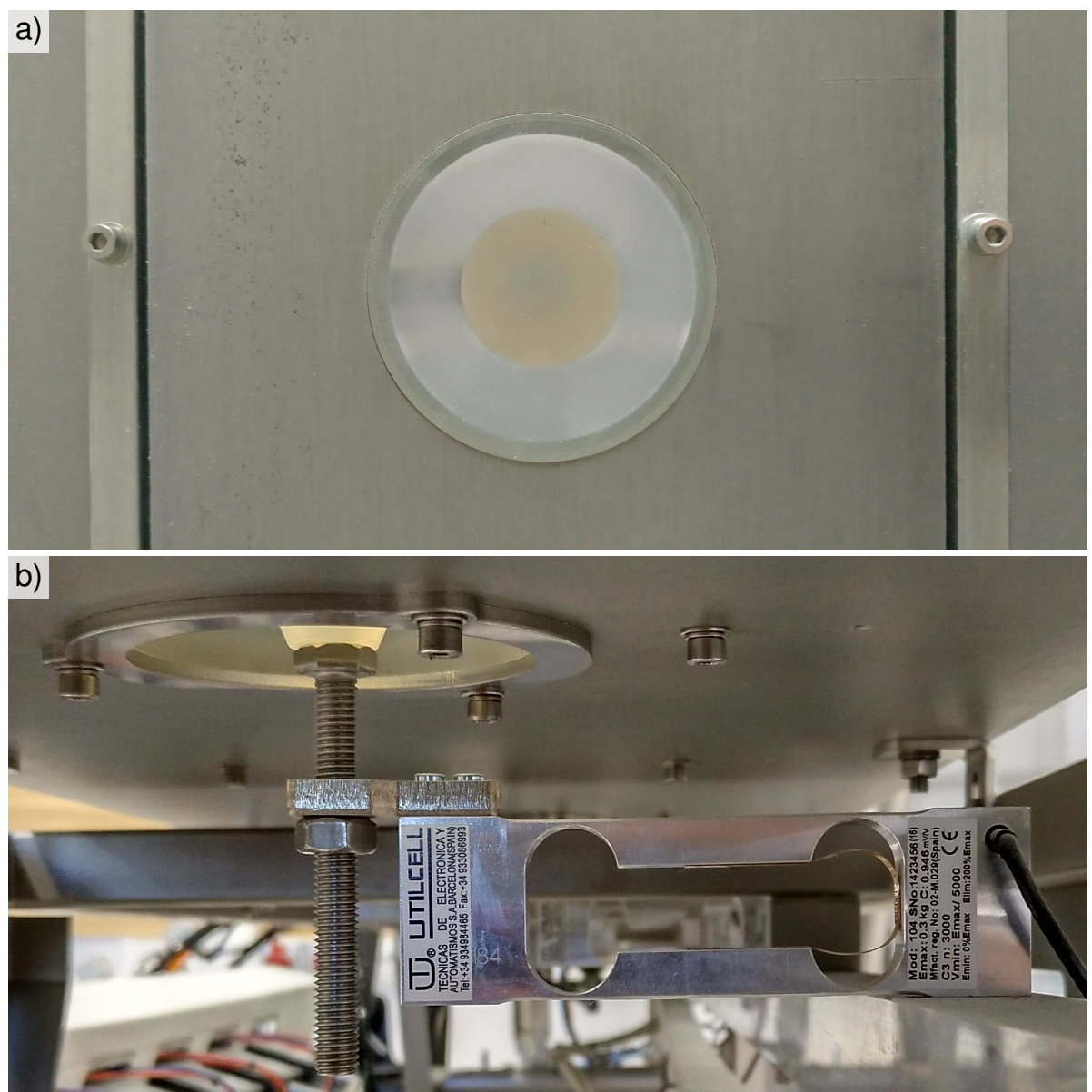

c)

$10 \mathrm{~mm}$

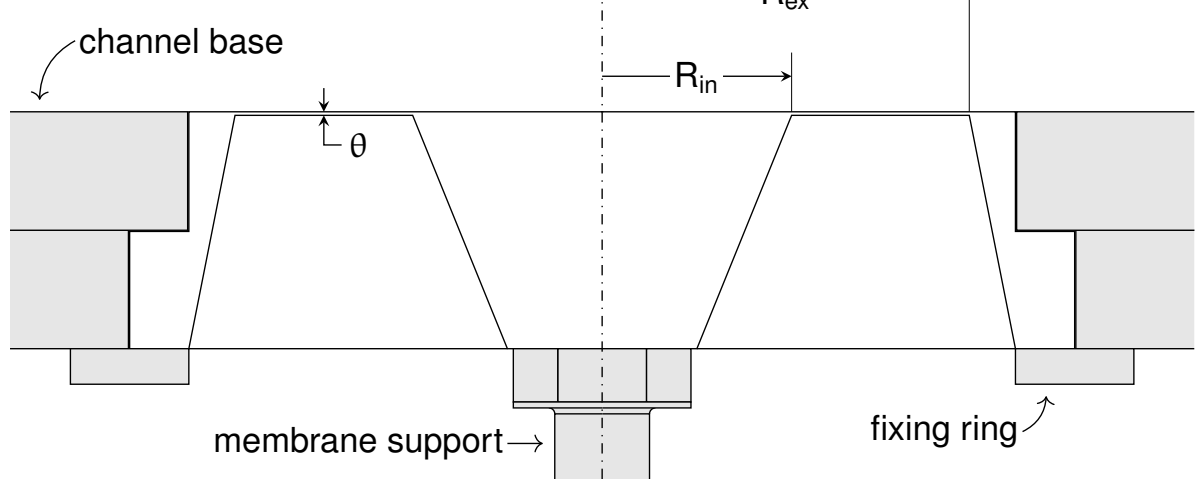

Fig. 5: Silicone membrane and load cell mounting: a) top view of the membrane embedded in the channel base; b) side view of the beam load cell arrangement with membrane support; c) diagram of the membrane design with internal radius $R_{\text {in }}=16 \mathrm{~mm}$ and external radius $R_{\mathrm{ex}}=31 \mathrm{~mm}$. 
12 for data acquisition and control by a custom interface of the linear guide, as well as the automated lifting gate, the reversible pneumatic circuit, and the signal filtering and transmission panel. The Gocator Web Interface by LMI Technologies is used to configure and control the profile sensor.

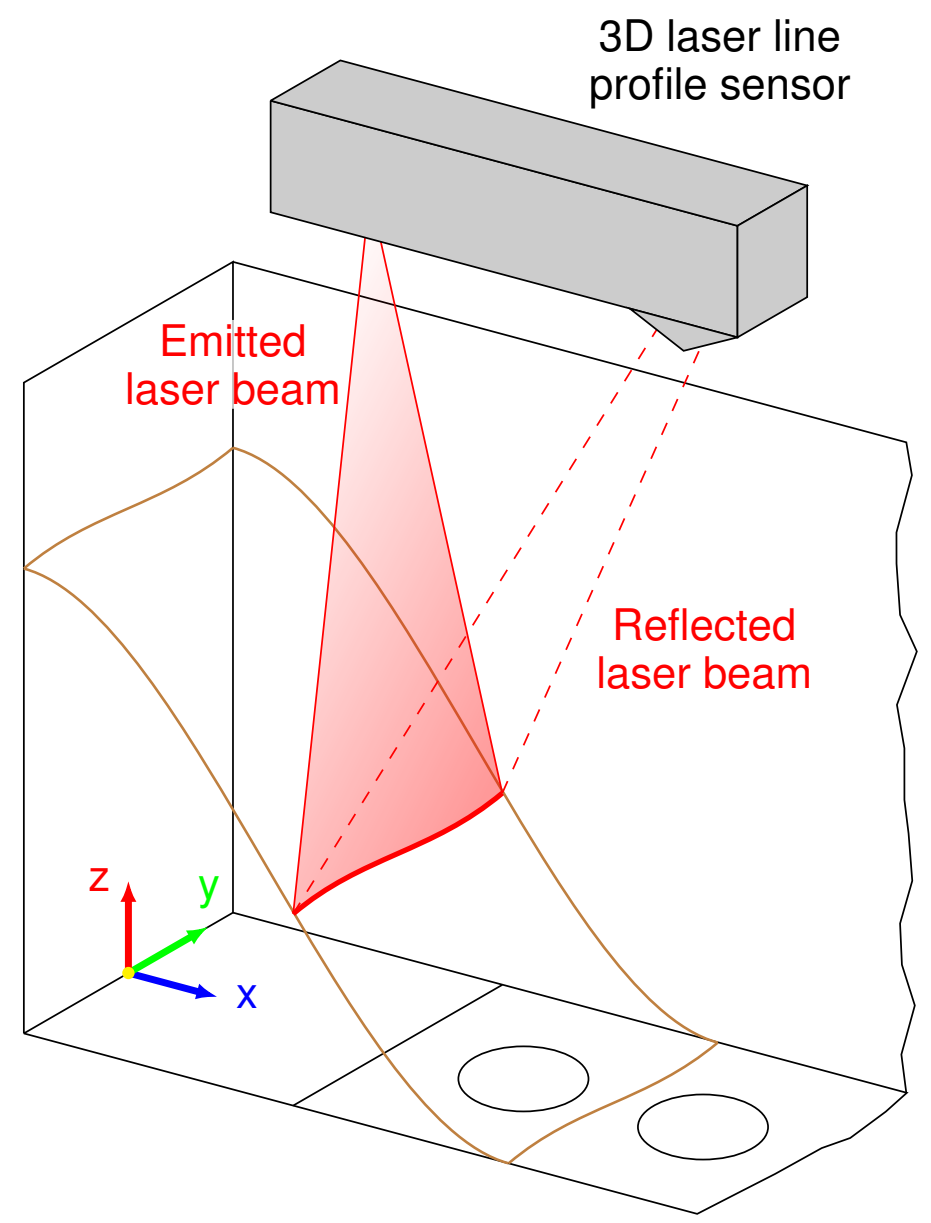

Fig. 6: Schematic view of the profile sensor positioning with respect to the frame of reference fixed on the channel base.

Near-wall flow visualisations through the glass walls of the channel are obtained using a high-speed video camera (SONY Corporation, Japan) at a frame rate of $200 \mathrm{fps}$ and with a pixel resolution of $1920 \times 1080 \mathrm{px}^{2}$. The digital imaging set includes a tripod (13) to ensure both the stability of the camera and the repeatability of the measurements, as well as two LED light panels (14) providing continuous lighting of the channel recordings. Kinematic fields 
are extracted from image analysis by particle image velocimetry (PIV) [56, a widespread technique for the study of steady-state flows down inclines in both dry [37, [57, 58] and saturated [59, 60] conditions. PIV analysis has been further incorporated to granular column collapse experiments [25, 26, 30, 40, 61 ] and similar set-ups [62. We use the open-source software PIVlab 63, which implements a cross-correlation algorithm performing iterative deformation and refinement of all interrogation areas in each frame pair being processed. Several approaches exist for image pre-processing to define object boundaries, as briefly reviewed in 64. Automatic image masking for background exclusion is required to delimit the region of interest, i.e. the flowing granular material; we ensure a uniform background by the chroma key in Fig. 2. Other moving objects in view throughout the recording, such as the lifting gate frame, are masked out by adapting the pattern matching scheme in the PIV to the object detection of reference images containing distinctive features. Granular materials able to produce particle texture above the pixel scale are properly tracked, whereas mixing the material with marker particles at low mass fraction may be required in the case of materials with insufficient inherent texture or with finer monochrome particles [53].

\section{Materials and testing protocols}

We have chosen three materials representative of common industrial practice to illustrate the capabilities of our experimental apparatus, namely: oat flakes, copper sulphate fertiliser, and talc powder. Table 1, shows material properties of the selected materials. We report poured bulk density $\rho_{\mathrm{p}}$ values corresponding

Table 1: Material properties of tested oat flakes, copper sulphate fertiliser, and talc powder samples.

\begin{tabular}{lccccccccccc}
\hline & \multicolumn{3}{c}{ Bulk density $\left(\mathrm{kg} \mathrm{m}^{-3}\right)$} & Friction & \multicolumn{2}{c}{ Particle } \\
Material & Poured & Aerated & Vacuum & Vibrated & coefficient & \multicolumn{2}{c}{$\begin{array}{c}\text { Circularity } \\
\text { size }(\mathrm{mm})\end{array}$} & $\begin{array}{c}\text { Gravimetric } \\
\text { coefficient }\end{array}$ & water content \\
& $\rho_{\mathrm{p}}$ & $\rho_{\text {aer }}$ & $\rho_{\text {vac }}$ & $\rho_{\text {vib }}$ & $\mu_{\mathrm{s}}(-)$ & $d_{10}$ & $d_{50}$ & $d_{90}$ & $C_{\mathrm{c}}(-)$ & $w(\%)$ \\
\hline Oats & 447 & 444 & 470 & 464 & $0.53-0.62$ & 1.45 & 2.66 & 3.94 & 0.63 & 12 \\
Fertiliser & 1136 & 1131 & 1142 & 1230 & $0.08-0.15$ & 0.105 & 0.185 & 0.353 & 0.76 & 1.2 \\
Talc & 455 & 427 & 530 & 466 & $0.4-0.8$ & 0.034 & 0.056 & 0.092 & 0.35 & 0.4 \\
\hline
\end{tabular}

225

to samples gently funnelled into the reservoir. The funnel is gradually lifted during filling to maintain a maximum distance of $150 \mathrm{~mm}$ between the release point 
and the surface of the accumulated material. The free surface of the resulting granular columns is manually levelled with a spatula, without exerting downward compaction of the poured samples. From the values of $\rho_{\mathrm{p}}$, the aerated $\rho_{\text {aer }}$ and vacuum $\rho_{\text {vac }}$ densities are measured by the volume change of the granular columns subject to air flows ranging between $\pm 2.5 \times 10^{-3} \mathrm{~m}^{3} \mathrm{~s}^{-1}$. The vibrated density $\rho_{\text {vib }}$ is obtained by gently pouring granular material samples into a measuring cylinder, to which a vibration is applied (of frequency $50 \mathrm{~Hz}$, maximum amplitude $0.3 \mathrm{~mm}$, and peak acceleration $40 \mathrm{~m} \mathrm{~s}^{-2}$ ) until volume change ceases. Additionally, we present values of the friction coefficient $\mu_{\mathrm{s}}$ for the oats from published results in a review of properties of seeds and grains in [65]; values for fertiliser are estimated from measurements on copper oxide films exposed to air tested at short reaction times after their formation in [66; ; and values for dry talc samples are also taken from experiments with slip distances of the order $10^{-2}$ to $1 \mathrm{~m}$ [67, at the same scale of our run-out observations. Furthermore, we extract the particle sizes of these powders and bulk solids from the sieve analyses presented in Fig. 7. Of the three materials, the oat flakes show the coarsest particle sizes up to $5 \mathrm{~mm}$. All the samples lie below the threshold value of $5 \%$ of the channel width, thereby confirming their testing suitability [18. Complementary material properties include: the circularity coefficient $C_{\mathrm{c}}$ describing the particle shape by image analysis, as the ratio of the projected particle area to that of a circle with the same perimeter [68]; and the gravimetric water content $w$ by oven-drying at $110{ }^{\circ} \mathrm{C}$ of samples at an ambient relative humidity of $54 \%$ and a room temperature of $20{ }^{\circ} \mathrm{C}$. In our view, the procedure to measure $w$ overestimates the amount of absorbed moisture on the oat flakes, by partially removing the adsorbed water in the organic constituents of the grains. Considering the conventional storage conditions of the three materials, we assume that our results illustrate the experimental investigation of dry granular flow.

We establish a testing protocol, valid for any granular material, to exploit all the redundant measurement systems incorporated to our column collapse apparatus, comprising the following steps:

Step 1. The lifting gate is closed and the sample is prepared by following the methodology described above to form a granular column of density $\rho_{\mathrm{p}}$ at an initial column aspect ratio $a \leq 2$. 


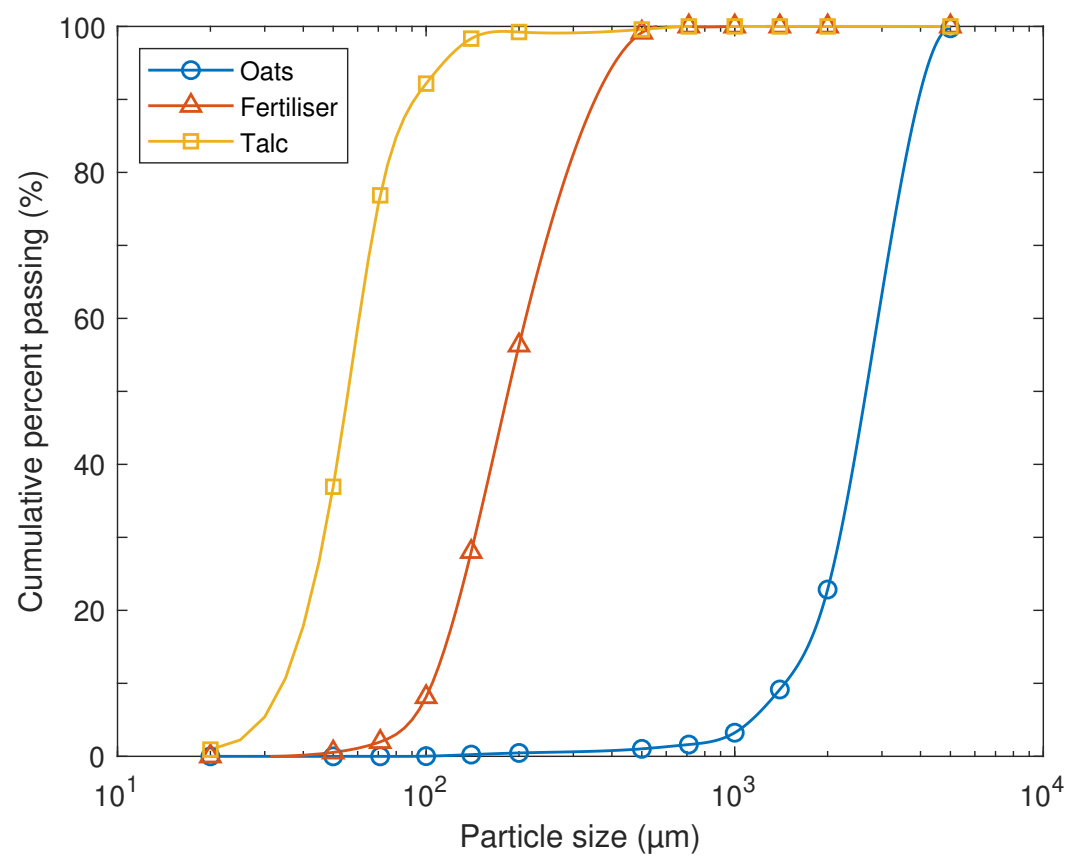

Fig. 7: Particle size distribution curves of tested oat flakes, copper sulphate fertiliser, and talc powder samples.

Step 2. The initial packing state is either as-prepared or pre-conditioned using one of the two operating modes of the reversible pneumatic circuit.

Step 3. Pre-conditioning is stopped and the profile sensor is used to scan the initial free surface of the granular column.

Step 4 . The high-speed video camera is manually triggered to capture the whole experiment during the camera recording time of $9 \mathrm{~s}$ at $200 \mathrm{fps}$. At the post-processing stage, we determine the start of our experiment when the difference of two consecutive frames first exceeds a threshold value. In the presented results, we have set threshold values at 1.2 to $2.0 \%$ of the peak frame difference throughout the videos, depending on the camera adjustments and the evolution of the experiment.

Step 5. Immediately after the high-speed video camera starts recording, a signal is sent that triggers both the lifting gate opening, and thus the onset of flow, as well as the data acquisition from the load cells, until the final 

to start at the time instants obtained by the procedure described in step 4. We delimit reaction times ranging from 134 to $247 \mathrm{~ms}$ that account for the lapse between the opening signal transmission and the activation of the pneumatic cylinders controlling the motion of the lifting gate.

Step 6. Once the flow stops, the lifting gate is completely detached from the channel to allow inspecting the final deposit, and the profile sensor is used again to measure the surface of the final deposit along the channel.

\section{Results and discussion}

In this section, we present selected results on three representative granular materials to display the main features of our experimental apparatus. We explore the pre-conditioning state of the granular columns by fluidisation and deaeration before collapse in subsection 4.1. We use the profile sensor to analyse the free surface morphology of the samples at rest, before and after collapse, to find out the run-out lengths and the slopes of the final deposits in subsection 4.2. The data gathered by the load cells enables us to study the load distribution at the channel base during flow propagation in subsection 4.3 . We also define the equilibrium flow heights as the relationship between the transmitted forces to the load cells and the height map measured by the profile sensor. In subsection 4.4 , we investigate the flow dynamics captured in the high-speed video recordings of the evolution of the near-wall height profiles, the flow front location, and the transient flow heights monitored jointly with the load cells. We deepen our analysis of flow kinematics in the last subsection 4.5 , by examining the PIV visualisations of the incremental displacement, velocity, and shear strain rate fields. This information allows determining the energy balance of the granular systems during the experiments.

Notice that, throughout this section, we nondimensionalise several physical quantities - including length, time, force, and energy - whenever we need to compare the findings from different tests. The versatility of our column collapse apparatus offers multiple combinations of testing steps, granular materials, and pre-conditioning of the samples, which have been considered for a thorough characterisation of flowability. Thereby, for the sake of clarity, we conveniently 
employ different characteristic quantities of interest in each nondimensional representation of our results.

\subsection{Initial packing state pre-conditioning}

Two different granular packing states result from initially poured samples at the random packing state. On the one hand, air fluidisation generates loose packing of the granular material. For fine-grained materials, an expansion behaviour is observed at air velocities above the minimum fluidisation value, which also involves gas channelling [69]. The initial column height without preconditioning $h_{\mathrm{p}}=128 \mathrm{~mm}$ of a talc sample is shown in Fig. $8 \mathrm{a}$ Air fluidisation provides an increase of the height with respect to $h_{\mathrm{p}}$ by $5.22 \%$, see Fig. $8 \mathrm{~b}$, resulting in the average initial column height after pre-conditioning $h_{0}=135 \mathrm{~mm}$. We observe the formation of preferential air flow paths, dominantly along the vertical direction, i.e. orthogonal to the minor principal stress direction. We also note the migration of fine particles at higher air velocities. On the other hand, deaeration produces dense packing of the granular material. The effect on the packing state is again greater for fine powders, which become densified under negative pressures preventing the formation of flow paths. However, as the column shrinks, some cracks open at the free surface of the sample. The average column height at the deaerated packing state $h_{0}=109 \mathrm{~mm}$ is observed in Fig. $8 \mathrm{~d}$ to be $15.1 \%$ shorter than the initially randomly packed granular column of height $h_{\mathrm{p}}=129 \mathrm{~mm}$ shown in Fig. $8 \mathrm{c}$

The air pressure drop across granular columns $\Delta P$ of fertiliser samples, as a function of the applied air flow $Q$, is depicted in Fig. 9. We observe that, as expected during fluidisation [70, the measured air pressures remain almost stable with the increase of air flow. The air pressure drop values are below the vertical stress at the reservoir base $\sigma_{z}$, due to self-weight of the poured column at random packing state

$$
\sigma_{z}=\rho_{\mathrm{p}} g h_{\mathrm{p}}
$$

where $g$ is the gravity acceleration. Vertical stresses range from 1.41 to $2.82 \mathrm{kPa}$ for columns of initial heights $h_{\mathrm{p}}$ between 124 and $254 \mathrm{~mm}$. Samples with larger initial column height correspond to higher air pressure drops, since air pressure accumulates towards balancing the body force. In the case of deaeration, the 

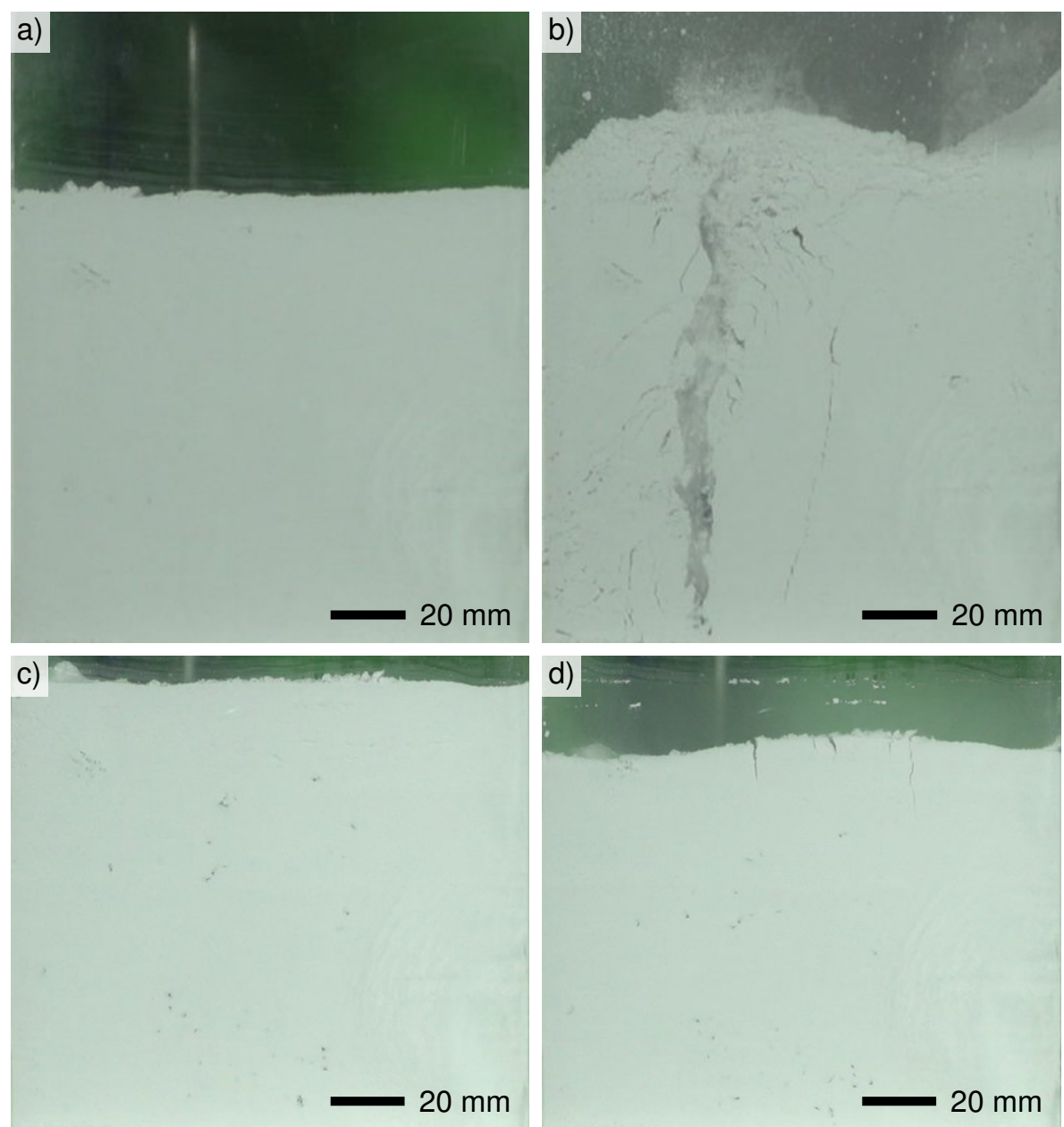

Fig. 8: Packing states of talc powder: a) initial random packing before fluidisation; b) fluidisation; c) initial random packing before deaeration; d) deaeration. 
absence of air flow paths explains the faster and more homogeneous distribution of the vacuum at lower negative air pressure values. At the same imposed air flow, larger negative air pressure drops are observed for columns with higher $h_{\mathrm{p}}$.

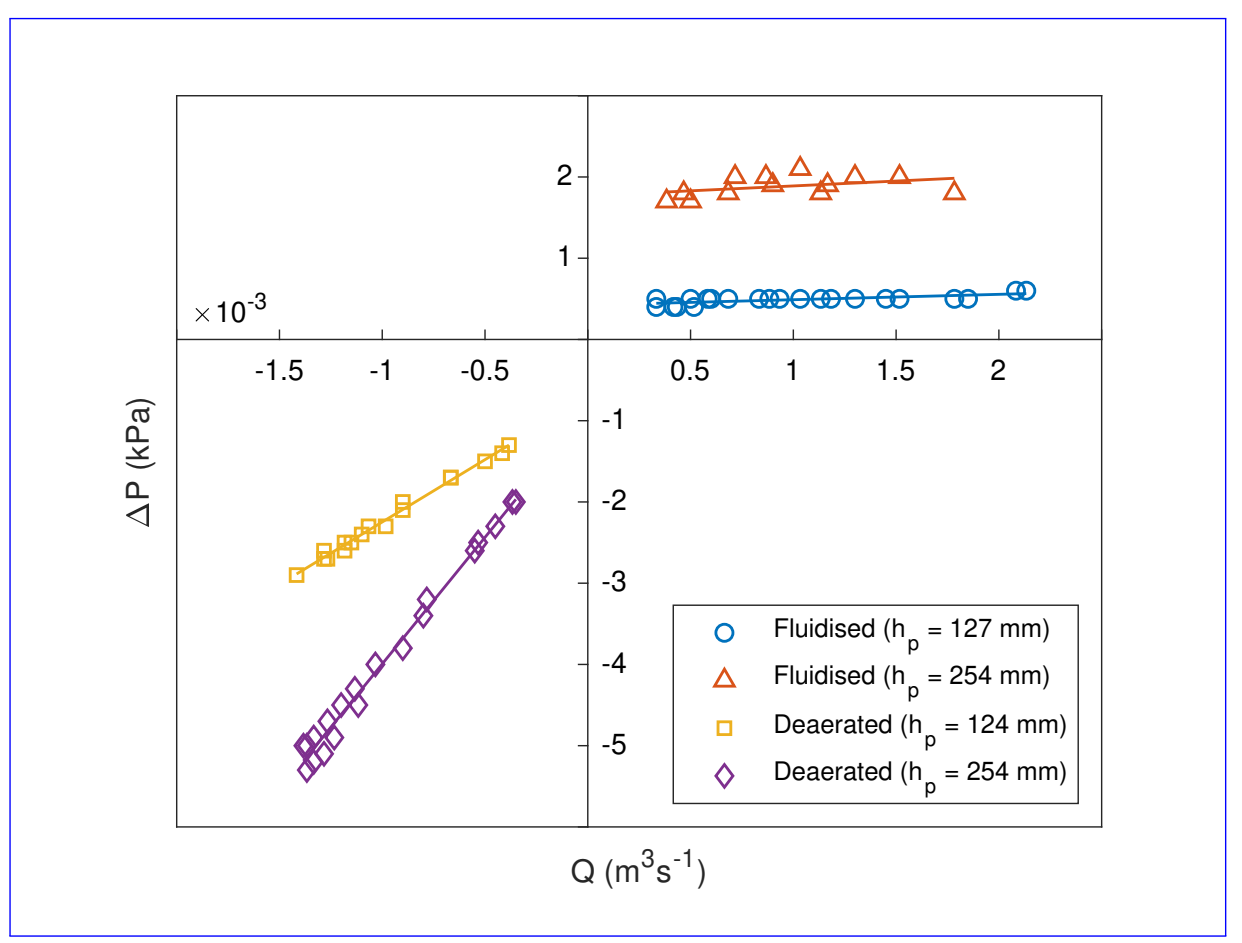

Fig. 9: Air pressure drop across fertiliser granular columns $\Delta P$ versus imposed air flow $Q$.

We use the data in Fig. 9 to derive the intrinsic air permeability $K$ of the fertiliser sample from the generalised Darcy's law

$$
\frac{Q}{A}=-\frac{K}{\mu_{\text {air }}}\left(\frac{\Delta P}{H}-\rho_{\text {air }} g\right)
$$

where $A$ is the cross-sectional area of the reservoir, and $H$ is the estimated average height, between $h_{\mathrm{p}}$ and $h_{0} . H$ is identified as the elevation head of the granular column, subject to a vertical air flux for the successive stationary stages of pre-conditioning, in terms of the air pressure and flow conditions. The air dynamic viscosity and the air density, respectively $\mu_{\text {air }}=1.82 \times 10^{-5} \mathrm{~kg} \mathrm{~m}^{-1} \mathrm{~s}^{-1}$ and $\rho_{\text {air }}=1.20 \mathrm{~kg} \mathrm{~m}^{-3}$, are measured at $20^{\circ} \mathrm{C}$ and $101 \mathrm{kPa}$. With maximum and minimum values as error bars, Fig. 10 shows the variation of $K$ as a function of 
porosity

$$
\varepsilon=1-\frac{m}{\rho_{\mathrm{s}} A H}
$$

where $m$ is the sample mass, and $\rho_{\mathrm{s}}=2.3 \mathrm{~kg} \mathrm{~m}^{-3}$ is the density of the fertiliser particles, resulting in an explored range of porosities from 0.49 to 0.58 . The air permeability of the fluidised states shows a large dispersion around the mean value $K=1.5 \times 10^{-10} \mathrm{~m}^{2}$, which is related to large pore distribution changes caused by the gas channelling and particle size segregation phenomena that take place for air flow values above the minimum fluidisation. On deaeration, the air permeability is around $K=4.6 \times 10^{-11} \mathrm{~m}^{2}$, roughly an order of magnitude smaller with respect to the fluidised samples, which we attribute to a reduction in the number of accessible pores in the densified granular columns.

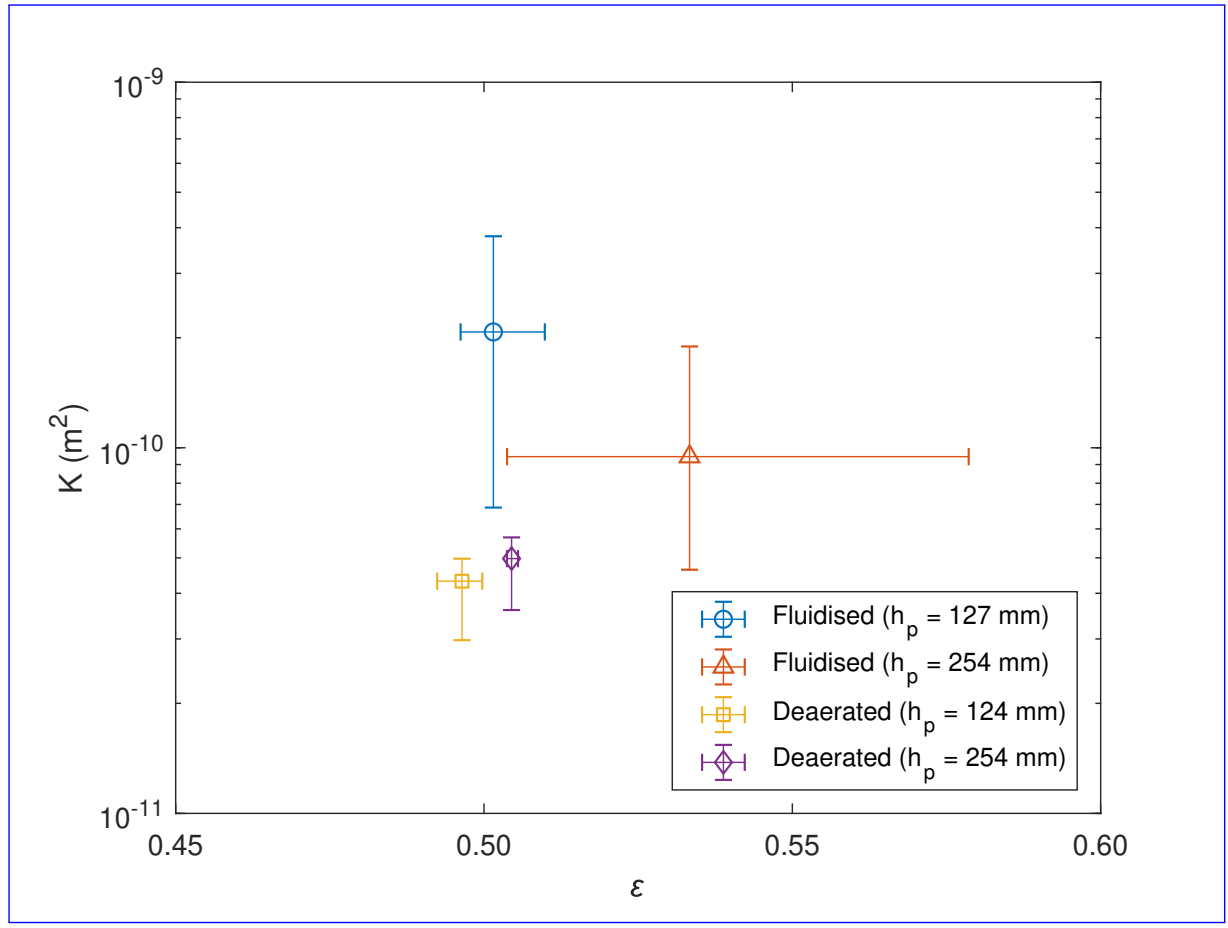

Fig. 10: Intrinsic air permeability $K$ versus average porosity $\varepsilon$ of the fertiliser samples.

\subsection{Free surface morphology and angle of repose}

Before the onset of flow, the initial free surface of the granular columns is scanned using the 3D laser line profile sensor. We use the generated height maps of points $\mathbf{x}=(x, y, z)$ to assess the average volume change of the samples 
corresponding to their packing state. Fig. 11 shows the nondimensional height variation of the free surface $-\Delta z / h_{\mathrm{p}}$ (compressive deformation is considered positive) of talc samples at the three packing states. Comparing the density measurements in Table 1, the compression response depends on the testing procedure and the material properties. Similar values of $\rho_{\mathrm{vac}}$ and $\rho_{\mathrm{vib}}$ are observed for the coarse oats samples. Fine-grained powders show larger compressions: fertiliser arranges into more compact packing states using vibration, with an average density increase by $8 \%$ with respect to $\rho_{\mathrm{p}}$; whereas deaeration provides the closest particle packing observed in the case of talc, see the average compression by $15 \%$ in Fig. 11c Regarding the effect of fluidisation, the fine-graded talc powder shows the largest average density reduction of the three materials, see the average expansion around $5 \%$ in Fig. 11a. The aerated density $\rho_{\text {aer }}$ of the other two coarser granular materials is reduced by $<1 \%$. As expected 69 , smaller expansion in comparison with the talc is observed for the oats considering its larger particle size (despite the similar $\rho_{\mathrm{p}}$ ), as well as for the fertiliser given its higher particle density.
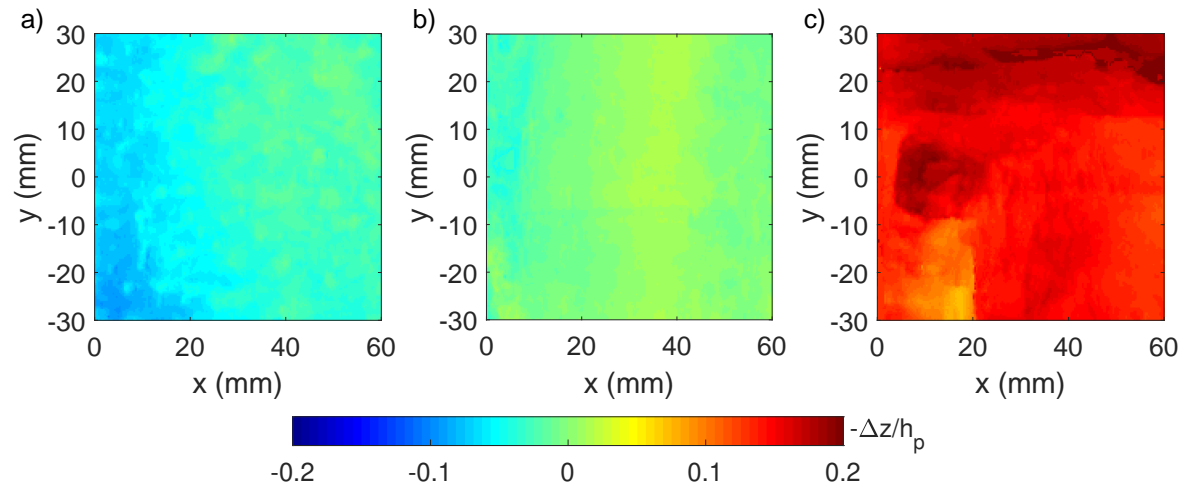

Fig. 11: Top views of the nondimensional height variation of the free surface $-\Delta z / h_{\mathrm{p}}$ of talc sample columns at different configurations: a) fluidised packing state $\left(h_{\mathrm{p}}=128 \mathrm{~mm}\right.$, $\left.h_{0}=135 \mathrm{~mm}\right)$; b) random packing state $\left(h_{\mathrm{p}}=h_{0}=127 \mathrm{~mm}\right)$; c) deaerated packing state $\left(h_{\mathrm{p}}=129 \mathrm{~mm}, h_{0}=109 \mathrm{~mm}\right)$.

At the end of our experiment, we measure again the free surface of the final deposits, as shown in Fig. 12. We take the initial volume of the granular columns into consideration by nondimensionalising the height and run-out length with respect to the initial column height after pre-conditioning $h_{0}$. This allows us 
to compare the observations of the different materials, based on their material properties. We observe that the maximum heights of the final deposits $h_{\infty}$ Fig. 1 are $h_{\infty} / h_{0}=0.8,0.7$ and 0.7 respectively for oats, fertiliser and talc samples. This indicates the complete collapse of the granular columns of initial column aspect ratio $a>1$. However, this behaviour is not always observed, especially in the case of samples with lower values of $a$ or that are pre-conditioned to a denser packing state, for which large fractions of the sample mass remain static during flow. Throughout the channel width, we observe on the one hand a lower variation of the height map in the case of fertiliser, see Fig. 12b a non-cohesive material with small particle sizes relative to the dimensions of our experimental apparatus. On the other hand, the free surface of the final deposit is more unevenly distributed along the $y$-axis for the cohesive talc powder and the oats, a material with coarser and more irregularly-shaped particles. We define the run-out length $l_{\infty}($ Fig. 1) as the average spreading of the tested samples. To this end, we use the average height profile of the final deposit in the $y$-axis direction $\bar{z}_{y}(x)$, taking into account the irregular distribution of the free surface along the channel width. We represent $l_{\infty}$ by vertical stripes located at the largest $x$-coordinate with $\bar{z}_{y}$ above the vertical resolution of the sensor. Consequently, we identify fertiliser as the material with larger spreading $l_{\infty} / h_{0}=2.3$ compared to the oats and talc samples, which show similar locations of the deposited flow front at $l_{\infty} / h_{0}=1.7$.

In Fig. 13a we compare $\bar{z}_{y}$ of an initially deaerated talc sample with a fluidised fertiliser sample. The height profile data is nondimensionalised in the $x$-axis and $z$-axis directions by the respective maximum values $l_{\infty}$ and $h_{\infty}$, to compare the height variation along the same unitary run-out. The gate opening generates a reduction of horizontal stresses in the granular material that causes collapse of the fluidised fertiliser sample. The deaerated talc column is nevertheless sustained by cohesion, where we observe the formation of a vertical cut at the final deposit configuration.

The angle of repose is a commonly used parameter in industrial practice [71, which we can recover from the slope of our final deposit measurements

$$
\alpha(x)=\tan ^{-1}\left(-\frac{\mathrm{d} \bar{z}_{y}}{\mathrm{~d} x}\right)
$$

as shown in Fig. 13b where the moving mean of $\alpha$ is represented using an 
a)

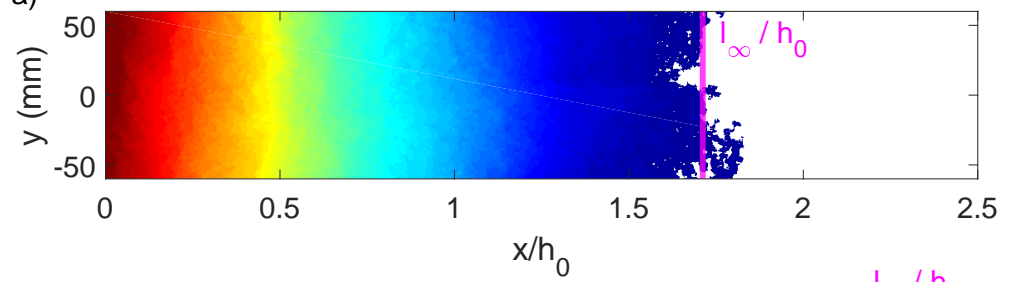

b)

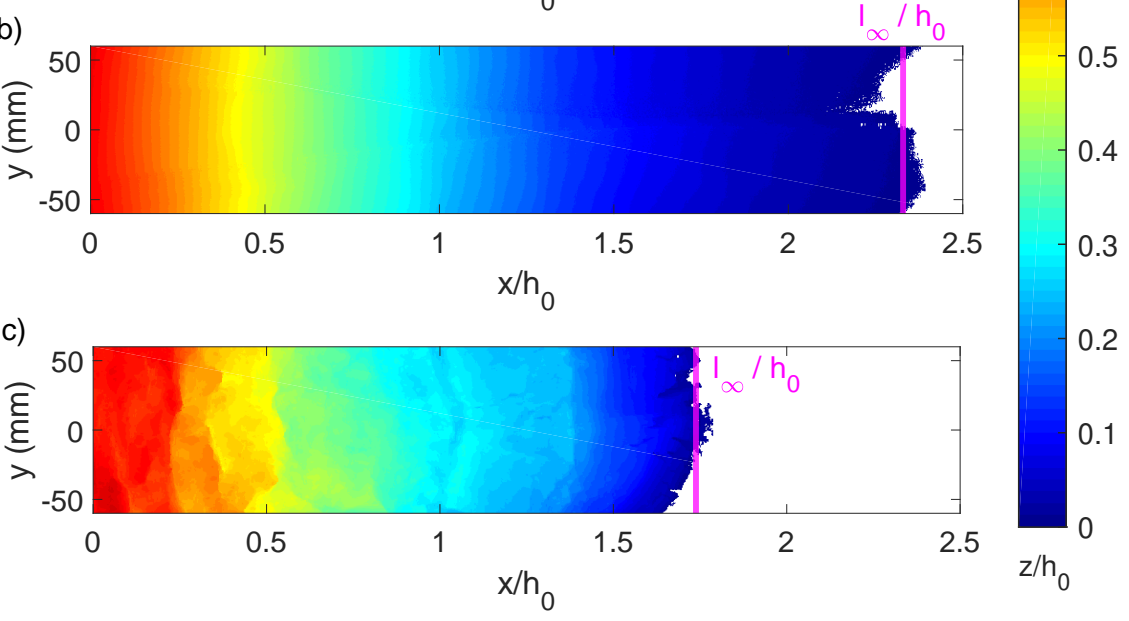

Fig. 12: Top views of the nondimensional free surface $z / h_{0}$ and runout lengths $l_{\infty} / h_{0}$ of three final deposits of tested samples without pre-conditioning: a) oats $\left(h_{0}=282 \mathrm{~mm}\right)$; b) fertiliser $\left.\left(h_{0}=253 \mathrm{~mm}\right) ; \mathrm{c}\right)$ talc $\left(h_{0}=243 \mathrm{~mm}\right)$. 
averaging length $20 d_{50}$ along the final deposit. The physical relevance of the angle of repose can be discussed by comparison with the friction coefficient $\mu_{\mathrm{s}}$, which governs the shearing mechanics of dry non-cohesive granular materials [54. The flow of fertiliser develops an angle of the final deposit below $28^{\circ}$ at the head and central parts of the deposit, gradually decreasing towards the run-out length to lower $\alpha$ values within the range of $\mu_{\mathrm{s}}$, see Table 1 . However, $\alpha$ of the deaerated talc sample shows peak angle values reaching $87^{\circ}$ around the vertical cut, and large $\alpha$ variations along the deposit length, which are not contained within the range of expected $\mu_{\mathrm{s}}$. These observations indicate that generally the angle also incorporates other physically relevant phenomena, including cohesion, besides the friction coefficient.

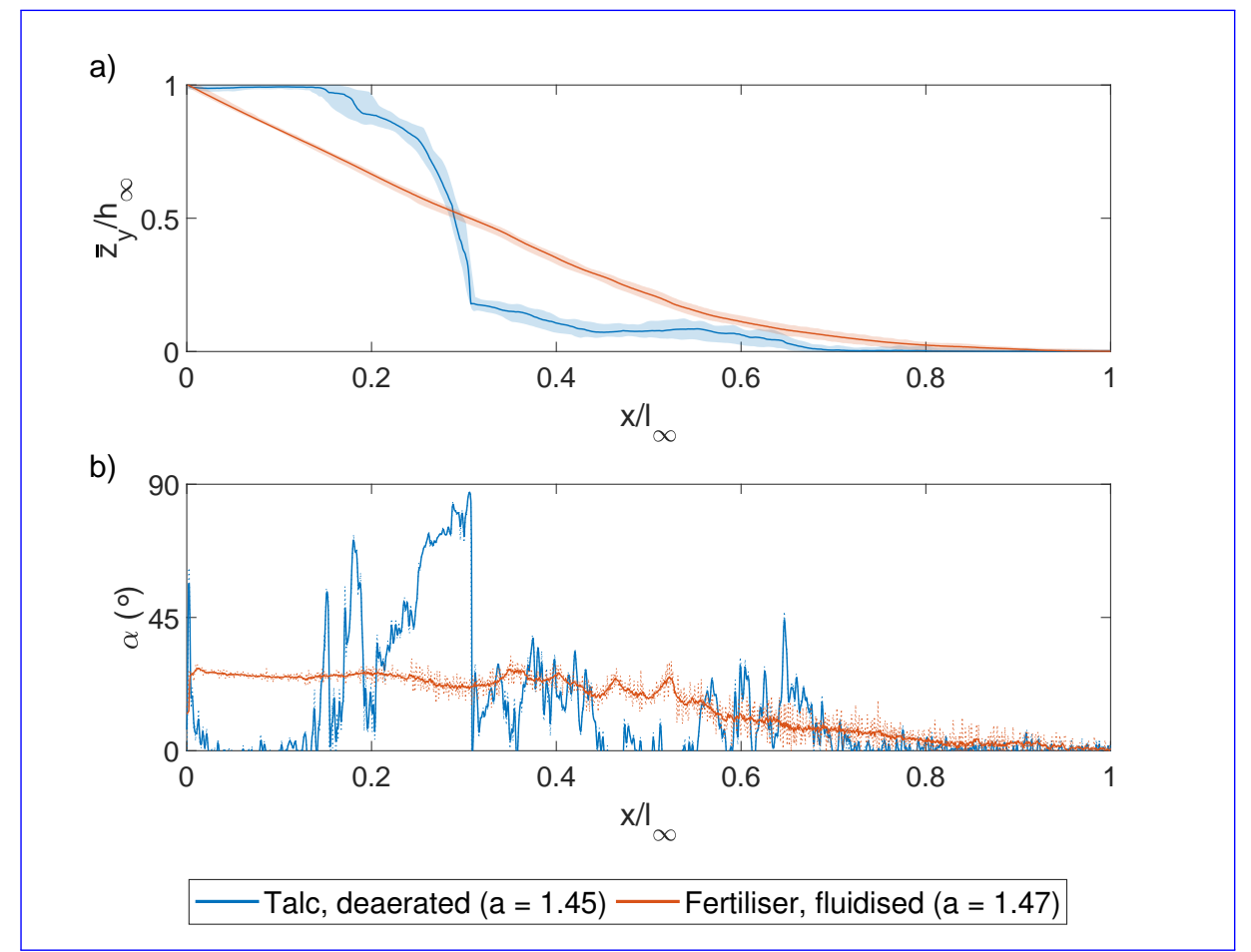

Fig. 13: Front views of two final deposit configurations of talc and fertiliser samples: a) nondimensional average height profile $\bar{z}_{y} / h_{\infty}$ along the nondimensional run-out length $x / l_{\infty}$ with shaded ranges of minimum to maximum $z(x)$; b) angle $\alpha$ along $x / l_{\infty}$.

\subsection{Basal load profiles and equilibrium flow heights}

We investigate the granular flow propagation from the load distribution on the channel base in time for the different samples represented in Fig. 15. We 
define the nondimensional load $\lambda\left(x_{k}, t\right)$ by the ratio of the force $f(t)$ measured by each transducer at the $x$-axis location $x_{k}, k=\{1, \ldots, 9\}$, to the weight force of the initial column $m g$

$$
\lambda\left(x_{k}, t\right)=\frac{f\left(\underline{x}_{k}, \underline{t}\right)}{m g}
$$

Moreover, we define the nondimensional time $\tau=t / t_{\mathrm{c}}$, where $t_{\mathrm{c}}=h_{\mathbb{Q}} \sqrt{g}$ is the characteristic time elapsed by a particle in free fall from an initial height $h_{0}$. Then, the experimental measurements obtained from the load cells $(\tau, \lambda)$ are fitted at each point $x_{k}$ by a nonlinear least squares criterion to the exponential model

$$
\lambda\left(x_{k}, \tau\right)=\lambda_{\text {ramp }}\left(x_{k}, \tau\right)+\lambda_{\text {peak }}\left(x_{k}, \tau\right)
$$

combining two Gaussian functions to explain the evolution of the basal loads in 440 time, see Fig. 14. On the one hand, the first summand in Eq. (6)

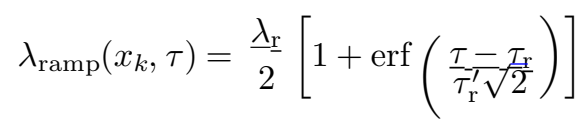

describes a load deposition ramp from the initial $\lambda=0$ to $\lambda=\lambda_{\mathrm{r}}$ at equilibrium, with three model parameters to be fitted: the basal load at equilibrium $\lambda_{\mathrm{r}}\left(x_{k}\right)$, the central ramp time $\tau_{\mathrm{r}}\left(x_{k}\right)$, and the ramp shape parameter $\tau_{\mathrm{r}}^{\prime}\left(x_{k}\right)$. We use the load deposition ramp model parameters $\tau_{\mathrm{r}}$ and $\tau_{\mathrm{r}}^{\prime}$ to estimate the times at which the ramp starts $\tau_{0}$ and ends $\tau_{\infty}$ by

$$
\tau_{0}=\tau_{\mathrm{r}}-3 \tau_{\mathrm{r}_{\infty}}^{\prime} \tau_{\infty}=\tau_{\mathrm{r}}+3 \tau_{\mathrm{r}}^{\prime}(8)
$$

as shown in Fig. 14. On the other hand, we observe an overshoot effect mainly for $a>1$ and sensed by the first force transducer, $\lambda_{\mathrm{p}}\left(x_{1}\right)$. Only in these cases we consider the second summand in Eq. (6)

$$
\lambda_{\text {peak }}\left(x_{k}, \tau\right)=\lambda_{\mathrm{p}} \exp \left[-\frac{1}{2}\left(-\bar{\tau}-\overline{\tau_{\mathrm{p}}^{\prime}} \tau_{\mathrm{p}}\right)^{2}\right]
$$

accounting for the occurrence of load peaks with three model parameters: the

${ }_{445}$ basal load peak $\lambda_{\mathrm{p}}\left(x_{k}\right)$, the central peak time $\tau_{\mathrm{p}}\left(x_{k}\right)$, and the peak shape parameter $\tau_{\mathrm{p}}^{\prime}\left(x_{k}\right)$. We have confirmed that the load peaks are negligible otherwise, since small values $\left|\lambda_{\mathrm{p}}\right|<10^{-4}$ are obtained in the fitting, and the physical interpretation of both $\tau_{\mathrm{p}}$ and $\tau_{\mathrm{p}}^{\prime}$ is lost.

Fig. 15 depicts the results of two representative trials of each experiment, ${ }_{450}$ and the average value of the fitted model parameters is detailed in Table 2. After 


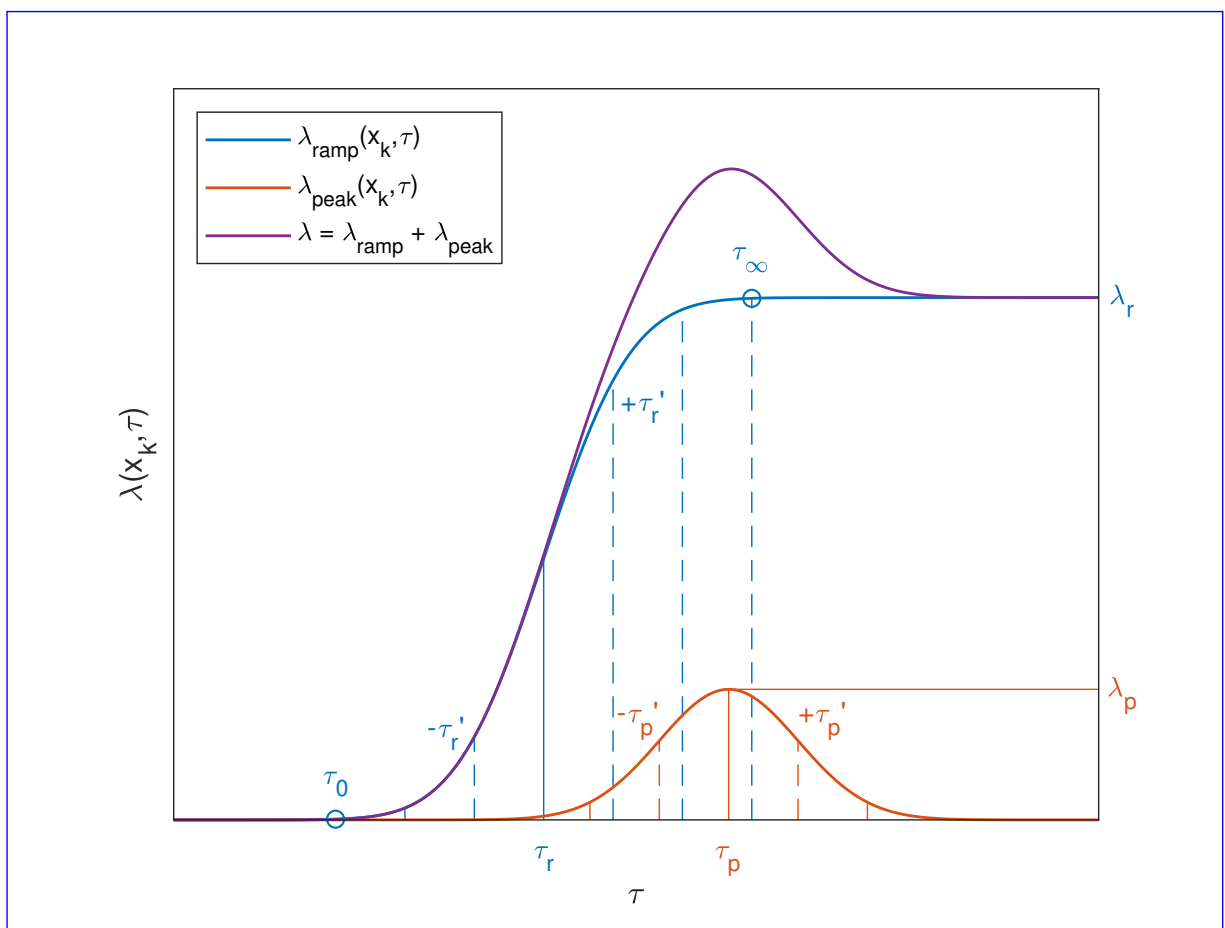

Fig. 14: Graph of the exponential model showing the combination of the two Gaussian functions $\lambda_{\text {ramp }}$ and $\lambda_{\text {peak }}$, respectively controlled by the model parameters $\lambda_{\mathrm{r}}, \tau_{\mathrm{r}}, \tau_{\mathrm{r}}^{\prime}$ and $\lambda_{\mathrm{p}}, \tau_{\mathrm{p}}, \tau_{\mathrm{p}}^{\prime}$. 
the onset of flow at $\tau=0$, the flow front propagates along the channel, reaching the first membrane at times $\tau_{0}\left(x_{1}\right)$ depending on the initial column aspect ratio: for $a<1$, fertiliser is the fastest advancing material and talc is notably delayed with respect to the other materials; for $a>1$, times $\tau_{0}\left(x_{1}\right)$ are reduced in average. Once the flow front advance is first detected at $\tau_{0}$, a depositional process develops at each load cell location on the channel base. During this process, loads increase monotonically at deposition rates up to around $\lambda_{\mathrm{r}} / \tau_{\mathrm{r}}^{\prime} \sqrt{2 \pi}$ at $\tau=\tau_{\mathrm{r}}$, until the final deposit at rest is formed after $\tau_{\infty}$ and loads stabilise around $\lambda_{\mathrm{r}}$. For $a<1$, maximum deposition rates between 0.010-0.012 are observed, whereas the maximum rates rise to values between $0.032-0.040$ as the initial column aspect ratio increases to $a>1$, showing similar values independently of the material. We notice comparable load profiles registered at the consecutive membrane positions, although with lower deposition rates at the successive $x_{k}$. The overshoot effect before load stabilisation is observed clearly for the fertiliser and talc samples, respectively showing load increments $\lambda_{\mathrm{p}}\left(x_{1}\right) / \lambda_{\mathrm{r}}\left(x_{1}\right)$ of $11 \%$ and $23 \%$ at peak times $\tau_{\mathrm{p}}\left(x_{1}\right)$ around the estimated $\tau_{\infty}\left(x_{1}\right)$. Close values of the peak shape parameter $\tau_{\mathrm{p}}^{\prime}\left(x_{1}\right)$ are found for both materials. Small negative load peaks are also captured by the model, which describe a smooth deceleration of the depositional process towards the equilibrium load $\lambda_{\mathrm{r}}$ albeit with a negligible effect on the basal load propagation trends. We interpret the load peaks to be caused by an initial flow stage of vertical collapse [25, 26, which generates the downward acceleration of the mobilised mass. This results in impulses exerted on the channel base at positions closer to the reservoir, that are in turn quickly dissipated as the motion becomes governed by the horizontal inertia of the propagating flow. Comparing the performance of the different materials, we observe that the talc powder at $a>1$ shows the largest $\lambda_{\mathrm{p}}\left(x_{1}\right)$, due to its abrupt vertical collapse, which also produces a steeper deposition rate on the second membrane. Talc shows the highest deviation in the attained values of $\lambda_{\mathrm{r}}\left(x_{k}\right)$, denoting an irregular distribution of flow front along the channel width, especially at the run-out configuration.

We identify a relationship between the basal load distribution registered at run-out and the free surface of the final deposit configuration. At equilibrium, the flow height $h_{k}$ at the $k$-th membrane location is determined by the balance 
Table 2: Nondimensional Gaussian model parameters fitted to average results at the load cell locations $x_{1}, \ldots, x_{4}$, of oats, fertiliser and talc samples of initial column aspect ratios $a$, tested at the random packing state.

\begin{tabular}{|c|c|c|c|c|c|c|}
\hline \multirow{2}{*}{ Materials } & \multirow{2}{*}{$a$} & \multicolumn{5}{|c|}{ Load cell location ( $\mathrm{mm}$ ) } \\
\hline & & & $x_{1}=225$ & $x_{2}=320$ & $x_{3}=425$ & $x_{4}=545$ \\
\hline \multirow{6}{*}{ Oats } & \multirow{3}{*}{0.93} & $\lambda_{\mathrm{r}}$ & 0.016 & 0.001 & & \\
\hline & & $\tau_{\mathrm{r}}$ & 2.78 & 3.97 & & \\
\hline & & $\tau_{\mathrm{r}}^{\prime}$ & 0.62 & 0.64 & & \\
\hline & \multirow{3}{*}{1.87} & $\lambda_{\mathrm{r}}$ & 0.024 & 0.013 & 0.003 & \\
\hline & & $\tau_{\mathrm{r}}$ & 1.79 & 2.76 & 3.29 & \\
\hline & & $\tau_{\mathrm{r}}^{\prime}$ & 0.24 & 0.54 & 0.52 & \\
\hline \multirow{12}{*}{ Fertiliser } & \multirow{6}{*}{0.85} & $\lambda_{\mathrm{r}}$ & 0.017 & 0.004 & & \\
\hline & & $\tau_{\mathrm{r}}$ & 2.31 & 3.57 & & \\
\hline & & $\tau_{\mathrm{r}}^{\prime}$ & 0.69 & 0.70 & & \\
\hline & & $\lambda_{\mathrm{p}}$ & -0.001 & - & & \\
\hline & & $\tau_{\mathrm{p}}$ & 2.99 & - & & \\
\hline & & $\tau_{\mathrm{p}}^{\prime}$ & 1.24 & - & & \\
\hline & \multirow{6}{*}{1.69} & $\lambda_{\mathrm{r}}$ & 0.024 & 0.013 & 0.005 & 0.001 \\
\hline & & $\tau_{\mathrm{r}}$ & 1.77 & 2.55 & 3.24 & 3.86 \\
\hline & & $\tau_{\mathrm{r}}^{\prime}$ & 0.29 & 0.65 & 0.63 & 0.56 \\
\hline & & $\lambda_{\mathrm{p}}$ & 0.003 & -0.001 & - & - \\
\hline & & $\tau_{\mathrm{p}}$ & 2.82 & 2.99 & - & - \\
\hline & & $\tau_{\mathrm{p}}^{\prime}$ & 0.48 & 1.06 & - & - \\
\hline \multirow{9}{*}{ Talc } & \multirow{3}{*}{0.86} & $\lambda_{\mathrm{r}}$ & 0.018 & & & \\
\hline & & $\tau_{\mathrm{r}}$ & 3.22 & & & \\
\hline & & $\tau_{\mathrm{r}}^{\prime}$ & 0.60 & & & \\
\hline & \multirow{6}{*}{1.63} & $\lambda_{\mathrm{r}}$ & 0.025 & 0.015 & 0.002 & \\
\hline & & $\tau_{\mathrm{r}}$ & 2.23 & 3.01 & 3.59 & \\
\hline & & $\tau_{\mathrm{r}}^{\prime}$ & 0.36 & 0.43 & 0.34 & \\
\hline & & $\lambda_{\mathrm{p}}$ & 0.006 & - & - & \\
\hline & & $\tau_{\mathrm{p}}$ & 3.14 & - & - & \\
\hline & & $\tau_{\mathrm{p}}^{\prime}$ & 0.69 & - & - & \\
\hline
\end{tabular}




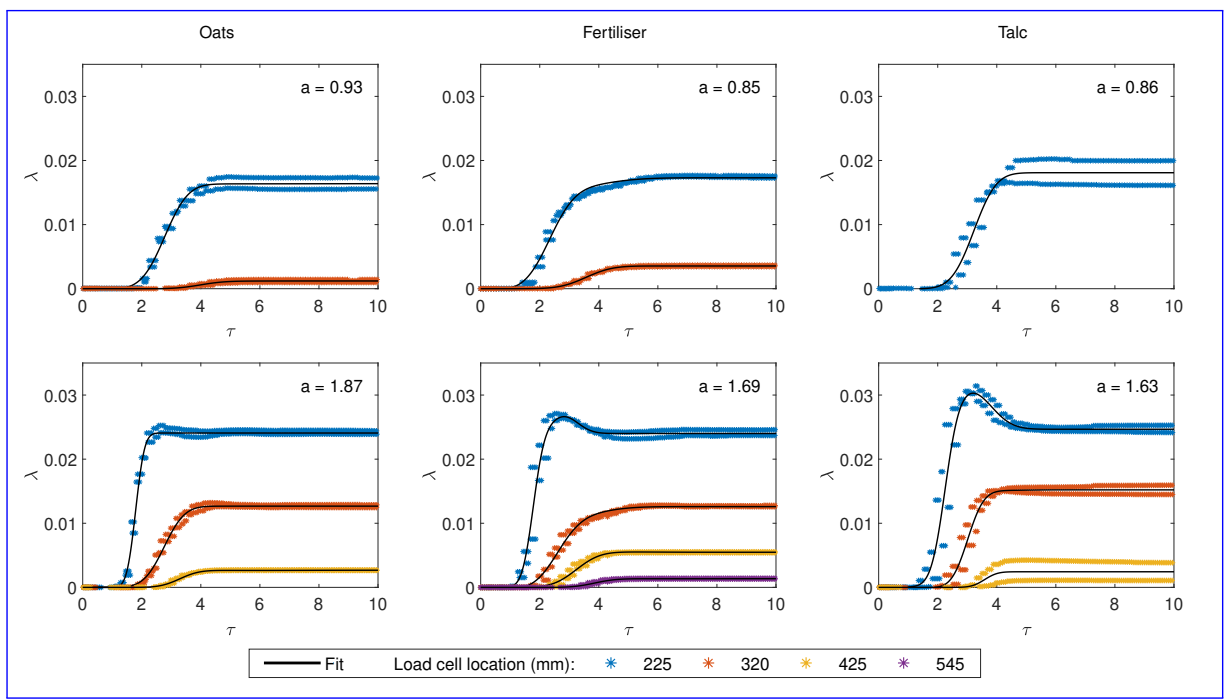

Fig. 15: Basal load profiles at the load cell locations $x_{1}, \ldots, x_{4}$ : nondimensional transmitted load $\lambda$ against nondimensional time $\tau$ for oats (left), fertiliser (centre) and talc (right) samples tested at the random packing state with varying values of $a$.

of forces between the load detected by the respective load cell $f\left(x_{k}\right)$ and the selfweight of an a priori unknown tributary volume of granular mass accumulated over the membrane. We assume a tributary volume consisting of a vertical cylinder of unknown radius $R_{k}$, expectedly between the minimum and maximum membrane radii $R_{\text {in }}$ and $R_{\mathrm{ex}}$, with a flat face on the channel base and the top face described by the free surface of the final deposit with average height $\bar{z}_{k}$. At the final static state, we take the flow height to be equal to the average height of the tributary volume, $h_{k}\left(\tau>\tau_{\infty}\right)=\bar{z}_{k}$, resulting in the balance of forces

$$
f\left(x_{k}\right)-\rho g \pi R_{k}^{2} h_{k}=0
$$

where $\rho$ is the time-dependent bulk density, evaluated by the volume change of the sample with respect to the initial granular column. We observe an overall increase of the bulk density of the final deposit by $1.9 \%$ with respect to the initial $\rho_{\mathrm{vac}}$ of the material. The results depicted in Fig. 16 show $R_{k}$ to lie around the midpoint between the membrane radii, with the calculated values ranging from 22.5 to $24.1 \mathrm{~mm}$. The observations systematically confirm the assumption on the tributary volumes for the calculation of the flow heights. Therefore, the measurements of the silicone membrane and load cell mountings can be verified at the final static state by means of the profile sensor system, thus benefiting 
from the instrumentation redundancy of our experimental apparatus.

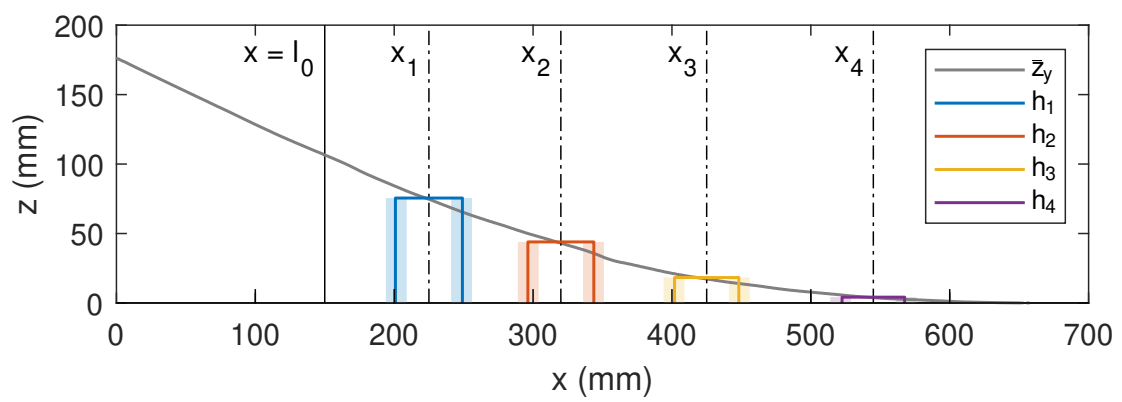

Fig. 16: Front view of the average height profile of the final deposit $\bar{z}_{y}$ and the flow heights $h_{1}, \ldots, h_{4}$ of an initially deaerated fertiliser sample $(a=1.69)$, where $\bar{z}_{k}$ are plotted as coloured lines spanning $R_{k}$ around the load cell locations $x_{k}$ and enclosed by shaded bars marking the range between the membrane radii $R_{\text {in }}$ and $R_{\text {ex }}$.

\subsection{Frontal flow visualisations and transient flow heights}

We study the frontal flow propagation along the rectangular channel to obtain a direct description of the granular motion close to the vertical glass wall from the high-speed video camera recordings. For all the tested samples, we accept the plane strain hypothesis on the granular flows, i.e. their quasi-twodimensionality, despite the uneven distribution of the free surface in the $y$-axis direction discussed in subsection 4.2. We interpret the near-wall results accordingly as representative of the overall behaviour within the channel.

To quantify the image analyses, first we perform the scaling of the video frames recorded by the high-speed camera. We use the calibrated profile sensor measurements of the initial granular column and the corresponding final deposit configuration. In Fig. 17, we establish a pixel scale of $0.58 \mathrm{px} \mathrm{mm}^{-1}$ by matching the near-wall height profile of the granular pile $z_{\text {wall }}$ to the free surface at the frontmost $y$-coordinate, $z\left(y_{\text {min }}\right)$.

In Fig. 18, we represent the evolution of $z_{\text {wall }}$ at the same instants in time $\tau$-nondimensional with respect to the characteristic time $t_{\mathrm{c}}$ of free fall from height $h_{0}$ - during the flow of two oats samples of initial column aspect ratios $a=0.93$ and 1.87 , tested at the random packing state. We use the characteristic lengths $l_{0}$ and $h_{0}$ for the nondimensional representation of the height profile 


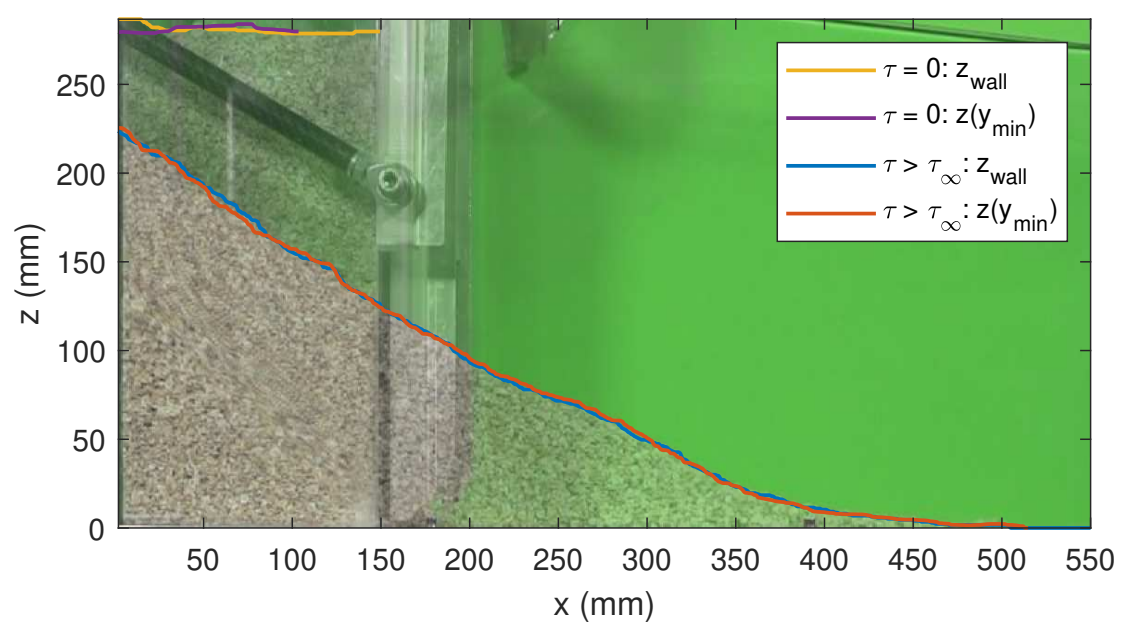

Fig. 17: Front views at the initial $(\tau=0)$ and final $\left(\tau>\tau_{\infty}\right)$ configurations of the nearwall height profiles of an oats sample tested without pre-conditioning, $z_{\text {wall }}$ and $z\left(y_{\min }\right)$, respectively obtained from high-speed camera and profile sensor measurements.

data in the $x$-axis and $z$-axis directions, respectively. This allows tracking the changes in the surface morphology starting from similar reference unitary regions. We distinguish three different stages of flow during propagation, starting immediately after collapse. In the first stage, the granular column deforms by maintaining a step-shaped free surface that advances in the $x$-axis direction. For $a<1$, we observe the free surface converging gradually towards a wedge-shaped surface for a time interval from $\tau=0$ to over $\tau=1.59$, see Fig. 18a. This initial stage of flow develops more rapidly for $a>1$, completing before $\tau=1.59$. Next, we detect a second stage of steady propagation of the flow front, which advances rapidly in regions closer to the channel base, until approximately $\tau=2.51$ in all cases. The height profile gradually develops into a wedge in the case of $a<1$, while the initial step-shaped configuration is preserved for $a>1$ until around $\tau=2.01$, as shown in Fig. 18b. Finally, in a third flow stage, the advance of the flow front stops at the run-out lengths $l_{\infty} / l_{0}=2.40$ and 3.37 for $a=0.93$ and 1.87 , respectively. In the final deposit configuration, we observe for $a<1$ that the granular material is only partially mobilised to an extent of approximately half the reservoir length, whereas for $a>1$ the complete collapse of the initial column takes place. 


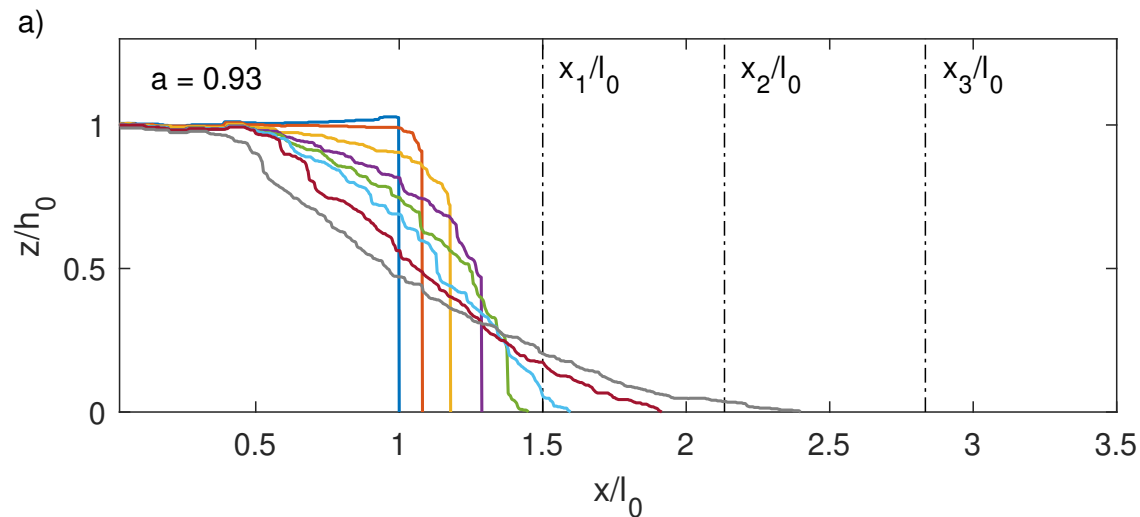

b)

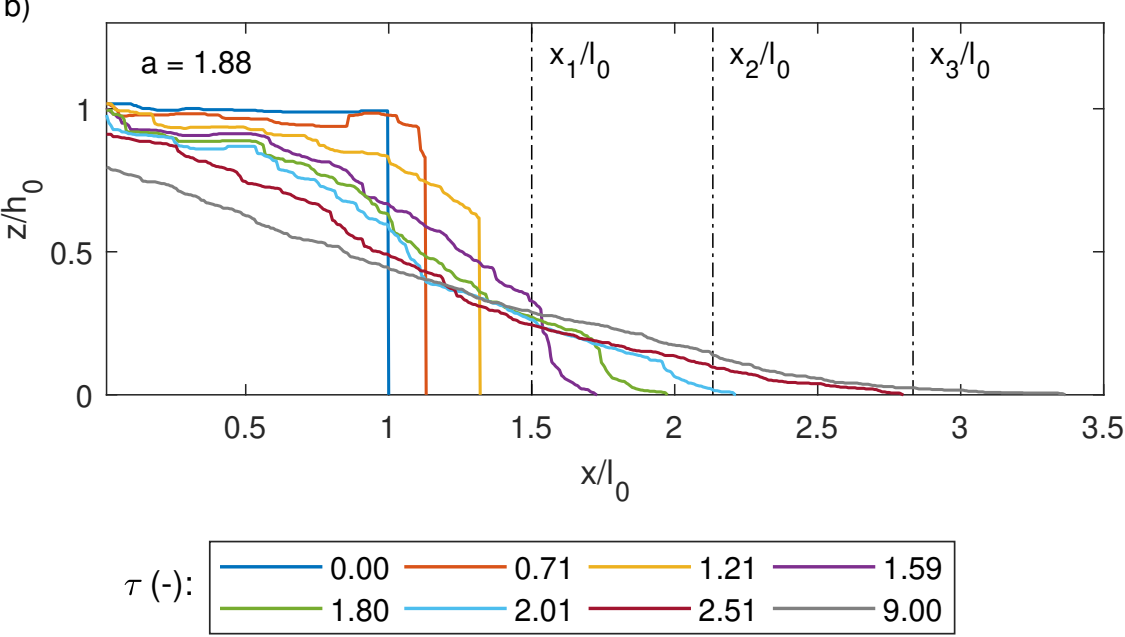

Fig. 18: Evolution in the nondimensional time $\tau$ of the nondimensional height profiles $z_{\text {wall }} / h_{0}$ of two oats samples tested at the random packing state with different values of $a$. 
Fig. 19 shows the location in time $\tau$ of the flow front $\phi$ of the tests depicted in Fig. 18, normalised by

$$
\phi(\tau)=\frac{x(\tau)-l_{0}}{l_{\infty}-l_{0}}
$$

to track the mobility reach of the flow front, evolving between the initial reservoir length $l_{0}$ and the final run-out length $l_{\infty}$. The image analysis results are contrasted with the basal load profile measurements, showing good agreement between the flow front trajectory and the load profile times $\tau_{0}\left(x_{k}\right)$-at which the $k$-th load cell locations are reached. The propagation of the flow front shows similar trends for the two oats samples at two different initial column aspect ratios. We observe an initial phase of slow propagation from $\tau=0.4$ to 1.5 , reaching $\phi=0.2$ in average, which corresponds to the first flow stage. Subsequently, we notice a phase of rapid propagation during the second flow stage, until the run-out length is reached at $\tau=4.2$.

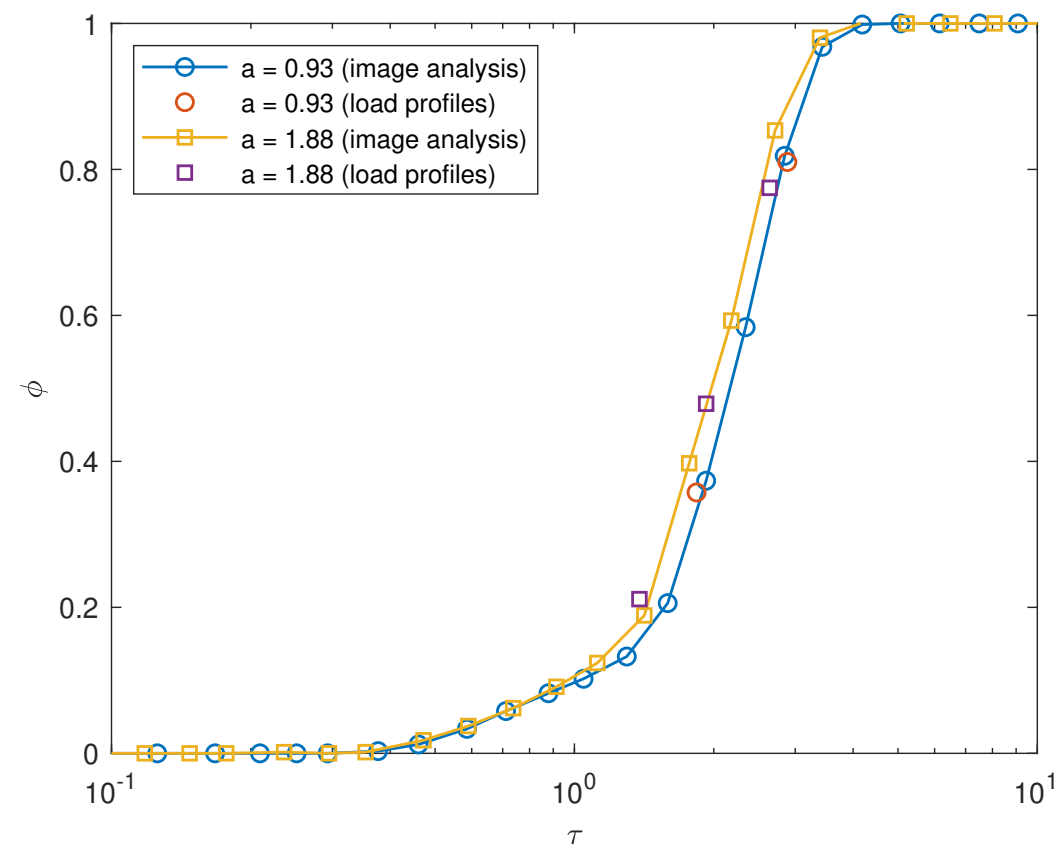

Fig. 19: Normalised flow front location $\phi$ in the nondimensional time $\tau$ of two oats samples of varying initial column aspect ratio $a$, tested at the random packing state.

Moreover, we use the basal load profiles as an estimate of the flow heights $h_{k}$ during the dynamic propagation of the granular material along the channel. 
In Fig. 20, we compare $h_{k}$ of the same deaerated fertiliser sample in Fig. 16 to the near-wall height profiles $z_{\text {wall }}$. At time $\tau=2.30$, we compute the flow heights $h_{k}(\tau)$ by the balance of forces in Eq. (10), assigning to the tributary radii $R_{k}$ the values calculated at the final static state of the sample, and using the instantaneous bulk density $\rho(\tau)$. An overall negligible decrease of the density by $0.6 \%$ is observed with respect to the initial $\rho_{\text {vac }}$. We observe that the transient flow heights $h_{k}$ do not correspond with the average profile heights $\bar{z}_{k}$. On the one hand, at the head of the wedge-shaped flow front, we find $h_{1}>\bar{z}_{1}$ by $29 \%$, which we correlate with the peaks observed in the basal load profiles, see Fig. 15. caused by the downward vertical acceleration of the mobilised mass due to the collapse of samples with $a>1$. On the other hand, we notice at the consecutive load cell locations that $h_{2}<\bar{z}_{2}$ and $h_{3}<\bar{z}_{3}$, respectively by 34 and $84 \%$. At the toe of the flow front, we observe that the propagation is dominated by the horizontal inertia of the flow, and hence the reason for the reduced flow heights.

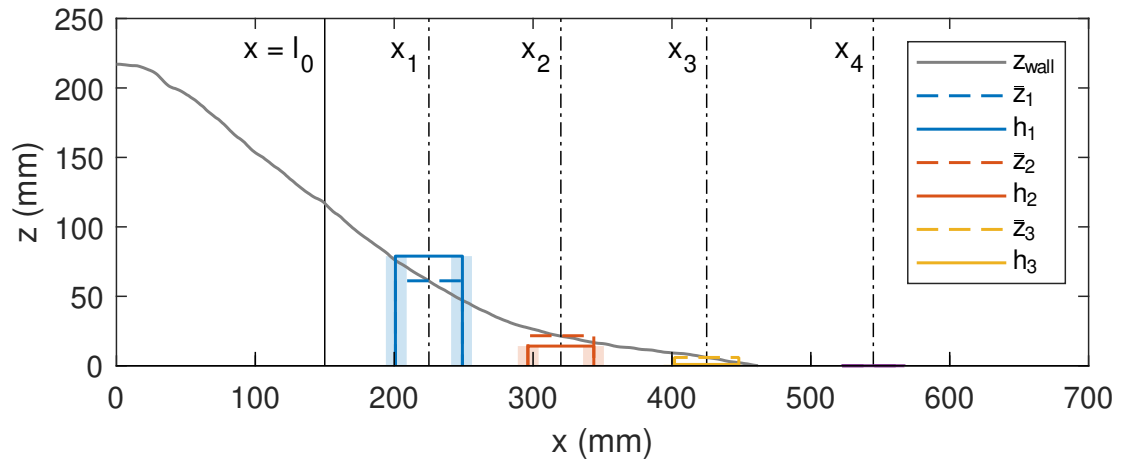

Fig. 20: Front view of the near-wall height profile $z_{\text {wall }}$ and the flow heights $h_{1}, \ldots, h_{3}$ at the recording time $\tau=2.30$ of the initially deaerated fertiliser sample $(a=1.69)$ depicted in Fig. 16 The average profile height $\bar{z}_{k}$ around the load cell locations $x_{k}$ is represented by dashed lines spanning $R_{k}$.

\subsection{PIV-based near-wall kinematics and energy balance}

We perform PIV analyses on the high-speed video recordings of the near-wall

flow propagation of the granular materials. The outcome of the post-process is the description of the incremental displacement field $\Delta \mathbf{u}=\left(\Delta u_{x}, \Delta u_{z}\right)$ between 
all pairs of consecutive video frames (recorded at $200 \mathrm{fps}$, i.e. with fixed time steps $\Delta t=0.005 \mathrm{~s}$ ), from which we derive the average velocity field in the elapsed time between frame pairs by $\mathbf{v}=\Delta \mathbf{u} / \Delta t=\left(v_{x}, v_{z}\right)$. In Fig. 21, we observe the near-wall kinematics of the flow of an oats sample of initial column aspect ratio $a=1.88$ by representing the velocity modulus

$$
|\mathbf{v}|=\sqrt{v_{x}^{2}+v_{z}^{2}}
$$

which shows the behaviour of mass mobilisation, alongside the absolute value of the incremental shear strain rate

$$
\left|\dot{\gamma}_{x z}\right|=\left|\frac{\partial v_{z}}{\partial x}+\frac{\partial v_{x}}{\partial z}\right|
$$

indicating the regions of localisation of the rate of change of the shear strains, at three representative time instants $\tau$ during flow. At $\tau=1.16$, we observe the displacement of an upper sliding block with respect to the static mass fraction at the bottom of the collapsing column, as shown in Fig. 21a During the first flow stage of deformation of the granular column due to collapse, the motion of the vertical flow front is dominated by large downward velocities showing peak values close to the free surface. The larger shear strain rates concentrate at the toe of the sliding block, as can be seen in Fig. 21b where we detect a localised shear band spreading over to the top free surface of the granular column. We notice two peaks of the velocity modulus at $\tau=1.90$, during the second flow stage of rapid propagation of the wedge-shaped flow front depicted in Fig. 21c one at the head of the wedge, where the downward velocities generate the avalanching of the granular material that is mobilised towards the toe; and the other peak located at the toe of the wedge with increasing horizontal velocities. The direction of the velocity field towards the toe of the wedge is gradually aligned with the horizontal surface of the channel base. Simultaneously, the shear strain rates represented in Fig. 21d develop closer to the free surface in comparison with observations at $\tau=1.16$. We notice large shear strain rates around the upper avalanching front and also at the lower part of the flow front. In Fig. 21e and Fig. 21f the third flow stage of deceleration of the flow is illustrated at $\tau=2.93$. The mass mobilisation slows down, as showed by the reduced values of the average velocity modulus and the incremental shear strain rate distributed close to the free surface. 

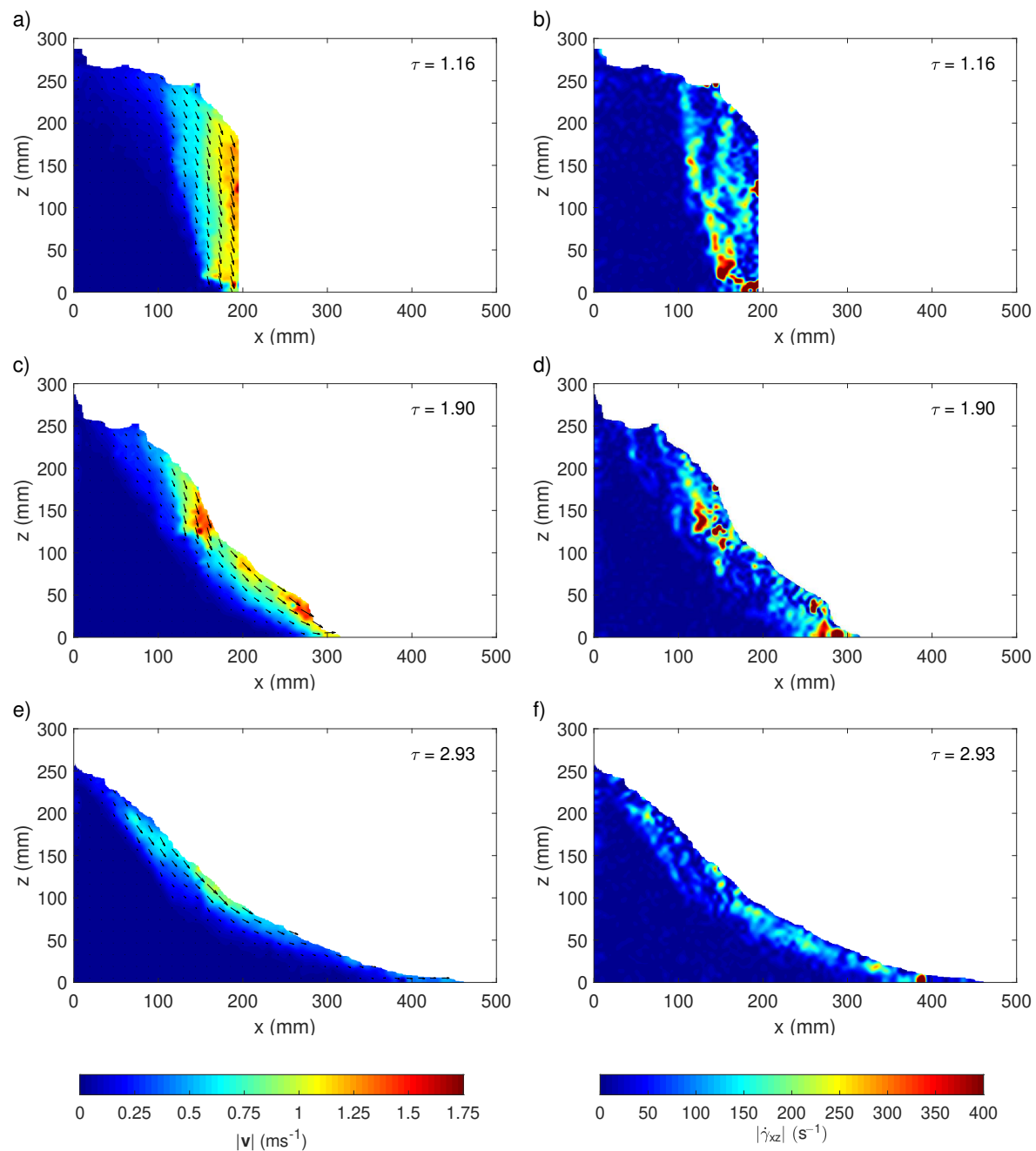

Fig. 21: Average velocity modulus (left) and absolute value of the incremental shear strain rate (right) fields at three different nondimensional time $\tau$ instants during the flow propagation of an oats sample $(a=1.88)$ tested at the random packing state. For reasons of readability, the color scales are saturated below the peak velocity $1.90 \mathrm{~m} \mathrm{~s}^{-1}$ and shear strain rate $925 \mathrm{~s}^{-1}$ at $\tau=1.90$ 
Fig. 22 shows the evolution in time $\tau$ of the maximum near-wall horizontal $v_{x}^{\max }$ and vertical $v_{z}^{\max }$ (with downward velocities positive for ease of representation) components of the velocity field during flow propagation of two oats samples alongside two fertiliser samples of varying initial column aspect ratios $a<1$ and $a>1$. All the samples are tested at random packing state. We observe an initially rapid increase of both maximum velocities during the first flow stage in the case of oats, followed by a deceleration of both maximum components. For the fertiliser, $v_{x}^{\max }$ continues to rise though slightly delayed with respect to $v_{z}^{\max }$. Both maximum components reach their peak values during the second flow stage, first $v_{z}^{\max }$ at a time $\tau<2$, followed by $v_{x}^{\max }$. We observe larger maximum vertical velocity peaks of the oats with respect to the fertiliser by $63 \%$ and $50 \%$, respectively for $a<1$ and $a>1$. For the oats, the peak values of $v_{x}^{\max }$ are $42 \%$ and $32 \%$ lower than the peak values of $v_{z}^{\max }$, respectively for $a<1$ and $a>1$, whereas similar peak values of both velocity components are obtained in the case of fertiliser. Afterwards, both velocity components decrease gradually during the last flow stage, until the final static state is reached by the oats sample. Velocities halt to a complete stop at $\tau<4$ for the fertiliser. In addition, we notice a greater dispersion of both velocity components in the oats samples throughout the flow. We explain this effect by a dominant avalanching mechanism in the flow, due to particle interlocking caused by the irregular shape of oat flakes. In contrast, the finer-grained fertiliser samples show a smoother evolution of the maximum velocity components. Our observations are in line with the schematic division of the flow dynamics originally proposed in 25, 26, as regards the description of the first two stages of flow. Although two flow stages are sufficient for the description of the flow dyamics of non-cohesive granular materials, such as fertiliser, we require adding a final third stage in the case of oats to describe the flow from when the run-out length is reached, by the end of the second flow stage, until the mass mobilisation ceases.

We analyse the granular flow behaviour from the point of view of the energy balance 72

$$
E_{\mathrm{tot}}=E_{\mathrm{pot}}+E_{\mathrm{kin}}+U
$$

where $E_{\text {tot }}$ is the total energy of the granular system, which is conserved, 
Oats
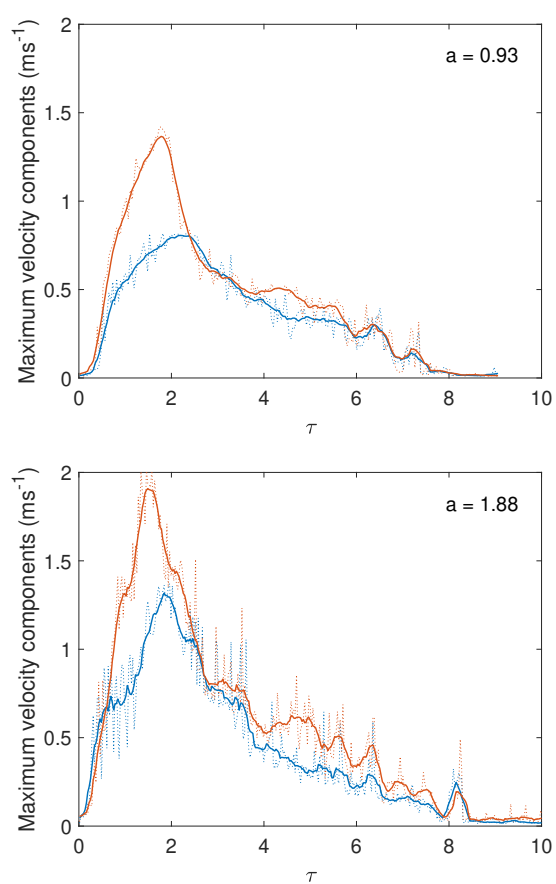

Fertiliser
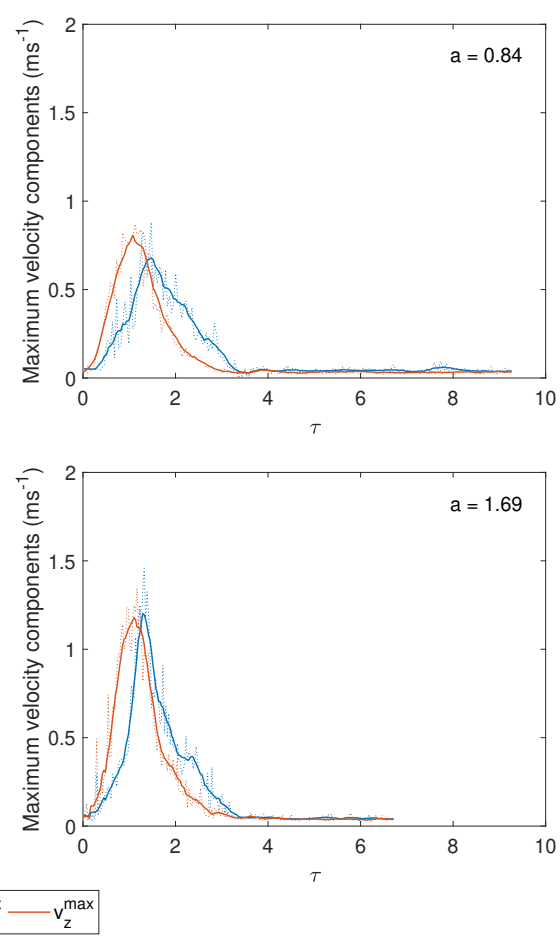

Fig. 22: Maximum near-wall horizontal $v_{x}^{\max }$ and vertical $v_{z}^{\max }$ components of velocity against the nondimensional time $\tau$ for oats (left) and fertiliser (right) samples tested at the random packing state with varying values of $a$. 
$E_{\mathrm{pot}}=m g z_{\mathrm{CM}}$ is the gravitational potential energy, where $z_{\mathrm{CM}}$ is the height of the centroid of the granular system, $E_{\text {kin }}=E_{\text {kin }}^{\text {trans }}+E_{\text {kin }}^{\text {rot }}$ is the kinetic energy, described by the mean square of the velocity fields resulting from the PIV analysis [62, and $U$ is the internal energy of the granular system, which accounts for the energy dissipated during flow. We compute $E_{\text {kin }}$ by the addition of the translational $E_{\mathrm{kin}}^{\text {trans }}=m \overline{\mathbf{v}^{2}} / 2$ and rotational $E_{\mathrm{kin}}^{\text {rot }}=I \overline{\boldsymbol{\omega}^{2}} / 2$ contributions, where $I \approx m d_{50}^{2} / 10$ is the moment of inertia of the granular system, approximated by the sum of the moments of inertia of all the particles assumed spherical, and $\boldsymbol{\omega}=\left(\partial v_{z} / \partial x, \partial v_{x} / \partial z\right)$ is the average angular velocity field in each time step. We use the near-wall height profile $z_{\text {wall }}$, and the average velocity fields $\mathbf{v}$ and $\boldsymbol{\omega}$, respectively to calculate the instantaneous $E_{\text {pot }}$, and $E_{\text {kin }}$. We normalise the energy components with respect to $E_{\text {tot }}$, allowing the response of the different materials to be compared. All energy components are redistributed in time $\tau$, as shown in Fig. 23 for two oats and two fertiliser samples, both with initial column aspect ratios $a<1$ and $a>1$, and tested at the random packing state. In all cases, we observe that $E_{\text {tot }}=E_{\text {pot }}$ at $\tau=0$, which is gradually transformed into the variations of $E_{\mathrm{kin}}$ and $U$ during flow of the granular material. We find a final decrease of $E_{\text {pot }}$ with respect to the initial values by 28 and $34 \%$ for $a<1$, and by 47 and $55 \%$ for $a<1$. The fertiliser samples show the larger reductions of the potential energy, which we explain by the greater spreading of the mobilised mass along the channel. Regarding $E_{\text {kin }}$, we notice that its variation is controlled by $E_{\text {kin }}^{\text {trans }}$, whereas we find the contribution of $E_{\text {kin }}^{\text {rot }}$ to kinetic energy to be negligible in the case of fertiliser, with peak values between 0.2 and $0.5 \%$ of the peak $E_{\text {kin }}^{\text {trans }}$. However, the peak $E_{\text {kin }}^{\text {rot }}$ values attain 4 and $13 \%$ of the peak $E_{\text {kin }}^{\text {trans }}$ for the oats samples, as expected for a granular material whose mass mobilisation is dominated by avalanching mechanisms, see Fig. 21. We notice a peak of $E_{\text {kin }}^{\text {trans }}$ at $\tau<2$, which we relate to the evolution of the instantaneous maximum velocity components shown in Fig. 22, reaching 11 and $14 \%$ of the respective $E_{\text {tot }}$ for the oats, and 2.6 and $3.0 \%$ in the case of fertiliser, larger for $a<1$ in both cases. Finally, in all cases, the increase of internal energy $U$ at the final static state is equal to the reduction of $E_{\text {pot }}$. According to [62], the continuous decay of $E_{\text {pot }}$ and the peak trend of $E_{\text {kin }}^{\text {trans }}$ suggest that the initial collapse (first flow stage) is dominated by gravity whereas flow propagation (sec- 
ond and third flow stages) is governed by dissipation processes. Consistently, we attribute the increase of $U$ mainly to irreversible particle interactions, including plastic normal collisions and the work of tangential forces and torques 73. As a result, the larger energy dissipation takes place for the fertiliser samples, which show greater mass mobilisation for all values of the initial column aspect ratio. Furthermore, our experimental evaluation of the model of energy balance in Eq. (14) is validated by our observations in Appendix A based on the numerical simulation results presented in [74].

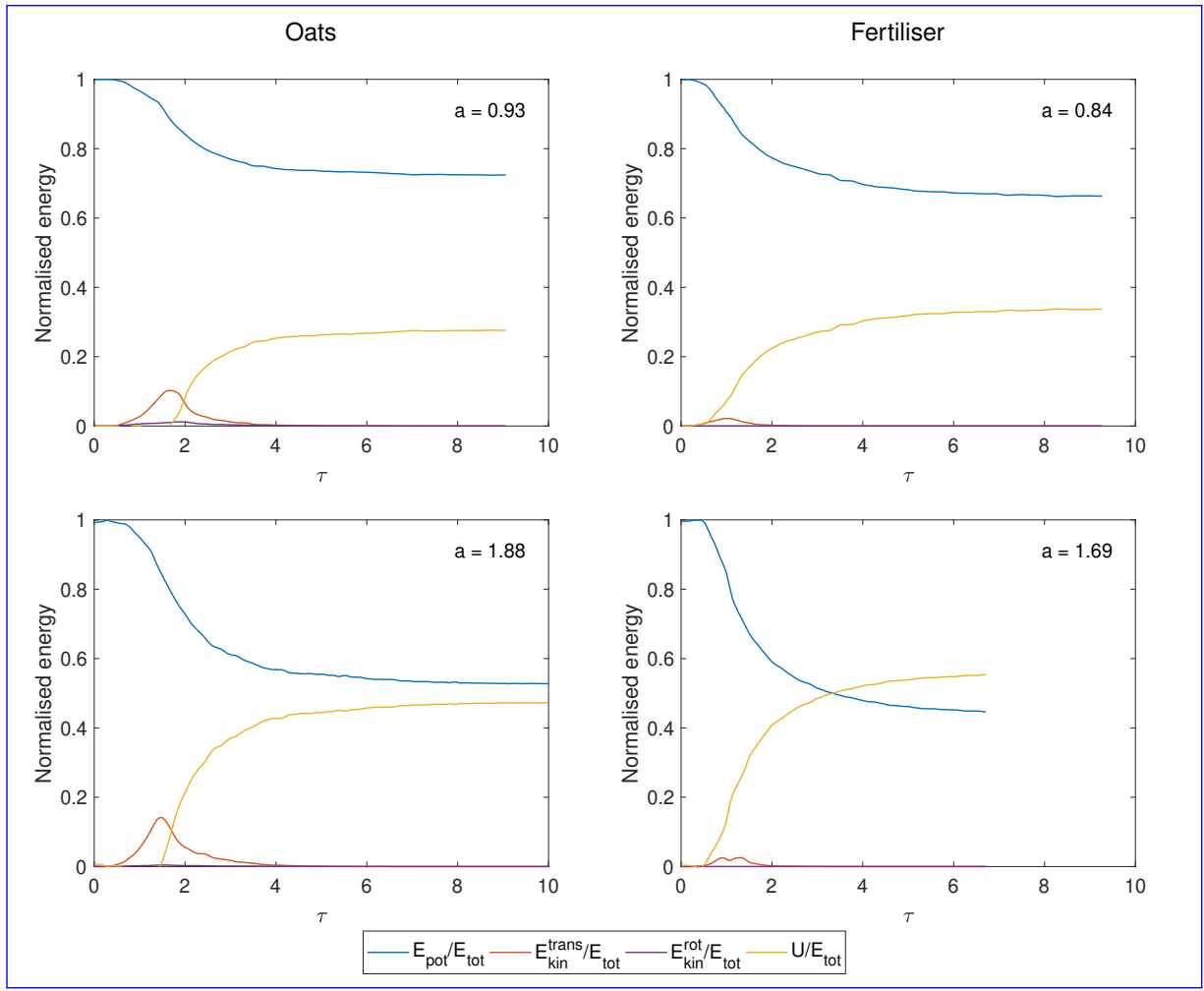

Fig. 23: Energy balance of the normalised potential $E_{\text {pot }}$, kinetic $E_{\text {kin }}^{\text {trans }}$ and $E_{\text {kin }}^{\text {rot }}$, and internal $U$ energies with respect to the total energy $E_{\text {tot }}$, during flow of oats (left) and fertiliser (right) samples tested at the random packing state with varying values of $a$.

\section{Summary and conclusions}

We have presented our new fully-instrumented granular column collapse apparatus responding to the need for an experimental methodology to characterise granular flow of a wide range of powders and grains. Our experimental set-up 
and the approach followed to interpret the results can be used as a reference framework to inform decision-making strategies for the adequate selection of bulk solids handling equipment, based on a quantitative evaluation of flowability of granular materials. Moreover, our experimental set-up can be further useful for granular flow modelling, with implications for the calibration of numerical model parameters, and the validation of the effect of the material properties and environmental conditions on flowability.

\subsection{Column collapse apparatus}

We have equipped our quasi-two-dimensional channel set-up with novel features and a diversity of redundant measuring techniques, which provide considerable insight into the flowability of powders and grains:

- A new lifting gate design, automated by a parallelogram mechanism that reduces the fast shearing and dragging effects on the samples during gate opening induced by existing vertical and swinging gates.

- A reversible pneumatic circuit with air flow and pressure control to study fluidisation and dearation effects on the material in granular column collapse set-ups. The air flow range of the circuit allows pre-conditioning the random packing state of the initial granular columns poured into the reservoir, reproducing actual handling conditions.

- An original system with silicone membranes and beam load cells to monitor the basal load distribution during flow at discrete positions along the channel surface. We adapt the weighing technology, employed for dosing in bag filling systems, to the analysis of flow propagation and deposition processes.

- A 3D line profile sensor controlled by a linear guide to scan the free surface morphology of the initial granular column and final deposit configurations of the tested samples. Laser scanning has been used previously on granular column collapse set-ups 35, and overcomes the measuring accuracy of other techniques, such as vernier scales with sliding laser pointers used in [23, 29]. 
- A high-speed video recording set, including a camera and LED lighting, to

5.2. Flow characterisation

We have demonstrated the capabilities of the apparatus with selected results from our granular flow tests with oat flakes, copper sulphate fertiliser, and talc powder samples, the highlights of which we summarise as follows:

- Fine-graded materials (fertiliser, talc) are pre-conditioned more effectively by our reversible pneumatic circuit. However, in the explored range of imposed air flow, neither full bed fluidisation nor significant vacuum compaction is attained for coarser materials (oats). In fluidisation conditions, the air pressure drop $\Delta P$ of powders reaches stabilisation due to the formation of preferential air flow paths, also influencing the variability of the intrinsic permeability $K$ of the material, whereas we observe a linear decrease of negative $\Delta P$ with the imposed vacuum, along with a reduction of $K$.

- We assume the plane strain hypothesis on the flow dynamics from the profile sensor measurements. The final deposits of fertiliser show a smooth slope and a larger run-out length $l_{\infty}$ with respect to the oats and talc samples. The talc flow presents the most uneven distribution of the mobilised mass - due to its cohesive nature - and shows a no-flow scenario when subjected to pre-conditioning by deaeration. We question the use of the angle $\alpha$ as a suitable flow descriptor for cohesive powders.

- We detect the basal load profiles with the set of load cells, which we fit to an exponential model describing a load deposition ramp $\lambda_{\text {ramp }}$ between times $\tau_{0}$ and $\tau_{\infty}$, and up to the equilibrium load $\lambda_{\mathrm{r}}$ at the final static state. 
For fertiliser and talc columns with initial column aspect ratio $a>1$, we observe the formation of a load peak $\lambda_{\text {peak }}$, which we attribute to the initial downward acceleration of the mobilised mass after collapse.

- We determine the flow heights $h_{k}$ from the basal load distribution in time. We contrast the flow heights with the profile sensor measurements of the free surface of the final deposit over the load cell locations $\bar{z}_{k}$ at equilibrium, and the transient near-wall height profiles $z_{\text {wall }}$ obtained by image analysis of the video recordings. The flowing fertiliser shows $h_{k}>\bar{z}_{k}$ at the cell locations closer to the head of the wedge-shaped flow front, coinciding with the development of $\lambda_{\text {peak }}$. We also find $h_{k}>\bar{z}_{k}$ at the load cell locations closer to the toe of the flow front, which we relate to its rapid propagation being dominated by the horizontal inertia of the spreading mass.

- We carry out PIV analyses of the video recordings to obtain the evolving near-wall kinematic fields (displacements, velocities, shear strain rates). We complement our visualisations with morphological data, comprising $z_{\text {wall }}$ and the flow front trajectory $\phi$. We use all this information to differentiate three stages describing the flow dynamics, for all values of $a$ :

- an initial flow stage dominated by the vertical component of velocity $v_{z}$, in which an upper sliding block is moving with respect to the material at rest at the bottom of the column, and thus explaining the mechanism leading to the registered $\lambda_{\mathrm{p}}$ in the basal load profiles;

- a second flow stage of rapid propagation of $\phi$ - described by the horizontal component of velocity $v_{z}$ at the toe of the wedge-shape flow front, and by $v_{z}$ at the head - which stops abruptly at length $l_{\infty}$;

- and a third and final flow stage of deceleration of the mass mobilisation, where both $v_{x}$ and $v_{x}$ are gradually reduced until the static final state is reached, which we clearly observe in the case of oats unlike for fertiliser.

- The energy balance of the granular system during flow propagation is controlled mainly by the redistribution of the potential energy $E_{\text {pot }}$ and 
the translational component of the kinetic energy $E_{\mathrm{kin}}^{\text {trans }}$, both of which we estimate from the image analysis of the frontal flow visualisations. We approximate the magnitude of the rotational component of the kinetic energy $E_{\text {kin }}^{\text {rot }}$, showing its little impact on the flow dynamics of the tested oats samples, and being almost negligible in the case of fertiliser. We attribute the increase in the internal energy $U$ to the dissipation generated by the interparticle contacts governing the mass mobilisation, this effect being more dominant for flows of fertiliser than in the case of oats. We validate the energy balance by DEM simulations.

In conclusion, we notice the good flowability of the copper sulphate fertiliser in that it is an easily aeratable material with a large mobility reach, as can be seen by the long run-out length and basal load distribution, exhibiting a flow propagation mechanism governed by frictional sliding in two stages of flow. The oat flakes show moderate flowability, having low sensitivity to both fluidisation and deaeration, and a flow dynamics controlled by avalanching related to particle interlocking that results into larger velocity peaks but an overall reduced mass mobilisation. We observe a poor flowability of the cohesive talc powder, which shows an uneven distribution of the deposited material in the static final state and a flow dynamics with short run-out lengths.

\subsection{Outlook}

We are currently investigating a methodology to predict the most suitable feeder type for the dosing of any given granular material by its classification into flowability clusters [75], defined by cluster analysis of a database of powders and grains. Conventional laboratory results, as well as the results from our experimental apparatus, provide us with key information to address this cluster analysis correctly. Therefore, the efficiency of the bulk dosing operations can be improved, yielding environmental and economic benefits for the packaging industry.

Future work will continue to explore the effect of particle shape non-sphericity using superquadrics [76], and the use of micro-macro transition methods [77] to calibrate and validate our DEM simulations against the experimental flow kinematics results from the PIV analyses. 


\section{Acknowledgements}

This work was supported by the Industrial Doctorates Plan of the Government of Catalonia [Project 2014 DI 075: Optimization of dosing systems for bulk solids using experimental and numerical techniques]. The authors would like to thank Joan Caba, Xavier Arderiu, Josep-Manel Padullés, and Juanjo González at TMI for their valuable contribution to the design and start-up of our experimental apparatus. The first author would like to acknowledge the support received by the Geotechnical Laboratory at the UPC in the early stages of this research.

\section{List of symbols}

Latin symbols

A Cross-sectional area of the granular column $\left(\mathrm{m}^{2}\right)$

a Initial column aspect ratio (-)

$C_{\mathrm{c}} \quad$ Circularity coefficient (-)

$d \quad$ Particle size by sieving, percent mass fraction passing in subscript (mm)

$E_{\text {kin }} \quad$ Kinetic energy of the granular system (J)

$E_{\text {kin }}^{\text {rot }} \quad$ Rotational kinetic energy of the granular system $(J)$

$E_{\text {kin }}^{\text {trans }} \quad$ Translational kinetic energy of the granular system $(\mathrm{J})$

$E_{\text {pot }} \quad$ Potential energy of the granular system $(\mathrm{J})$

$E_{\text {tot }} \quad$ Total energy of the granular system $(\mathrm{J})$

$f \quad$ Force transmitted to the load cells $(\mathrm{mN})$

$g \quad$ Acceleration of gravity $\left(\mathrm{m} \mathrm{s}^{-2}\right)$

$H \quad$ Average height of the granular column during pre-conditioning ( $\mathrm{mm}$ )

$h_{\infty} \quad$ Maximum height of the final deposit ( $\mathrm{mm}$ )

$h_{0} \quad$ Initial height of the granular column after pre-conditioning, if any (mm)

$h_{k} \quad$ Flow height at the $k$-th membrane location (mm)

$h_{\mathrm{p}} \quad$ Initial height of the granular column at the random packing state $(\mathrm{mm})$

I Moment of inertia of the granular system $\left(\mathrm{kg} \mathrm{m}^{2}\right)$

$K \quad$ Intrinsic air permeability $\left(\mathrm{m}^{2}\right)$ 
$l_{\infty} \quad$ Run-out length of the final deposit (mm)

$l_{0} \quad$ Reservoir length $(\mathrm{mm})$

$m \quad$ Sample mass $(\mathrm{kg})$

$Q \quad$ Air flow $\left(\mathrm{m}^{3} \mathrm{~s}^{-1}\right)$

$R_{\text {ex }} \quad$ External radius of the membranes ( $\mathrm{mm}$ )

$R_{\text {in }} \quad$ Internal radius of the membranes $(\mathrm{mm})$

$R_{k} \quad$ Radius of the cylindrical tributary volume of granular mass over the $k$-th membrane (mm)

$t \quad$ Time (s)

$t_{\mathrm{c}} \quad$ Characteristic time $(\mathrm{s})$

$U \quad$ Internal energy of the granular system $(\mathrm{J})$

$\mathbf{v} \quad$ Average velocity field $\left(\mathrm{m} \mathrm{s}^{-1}\right)$

$w \quad$ Gravimetric water content (\%)

$x_{k} \quad$ Location of the load cells on the $x$-axis, $k=\{1, \ldots, 9\}(\mathrm{mm})$

$y_{\min } \quad$ Frontmost $y$-coordinate of the channel width $(\mathrm{mm})$

$z_{\mathrm{CM}} \quad$ Height of the centre of mass of the granular system (m)

$\bar{z}_{k} \quad$ Average height of the free surface of the final deposit over the $k$-th membrane location ( $\mathrm{mm}$ )

$z_{\text {wall }} \quad$ Near-wall height profile of the granular pile $(\mathrm{mm})$

$\bar{z}_{y} \quad$ Average height profile of the final deposit in the $y$-axis direction $(\mathrm{mm})$

Greek symbols

$\alpha \quad$ Angle of the final deposit $\left(^{\circ}\right)$

$\dot{\gamma}_{x z} \quad$ Incremental shear strain rate $\left(\mathrm{s}^{-1}\right)$

$\Delta P \quad$ Air pressure drop across the granular column ( $\mathrm{kPa})$

$\Delta t \quad$ Fixed time step between consecutive video frames (s)

$\Delta \mathbf{u}$ Incremental displacement field between consecutive video frames (m)

$\Delta z \quad$ Height variation of the free surface of the granular column after preconditioning $(\mathrm{m})$

$\varepsilon \quad$ Average porosity (-)

$\theta \quad$ Membrane thickness ( $\mathrm{mm})$

$\lambda \quad$ Nondimensional transmitted load (-) 
$\lambda_{\mathrm{p}} \quad$ Nondimensional peak load of the basal load profile peak model (-)

$\lambda_{\text {peak }}$ Nondimensional basal load profile peak model (-)

$\lambda_{\mathrm{r}} \quad$ Nondimensional equilibrium load of the basal load profile ramp model

$(-)$

$\lambda_{\text {ramp }} \quad$ Nondimensional basal load profile ramp model (-)

$\mu_{\text {air }} \quad$ Air dynamic viscosity $\left(\mathrm{kg} \mathrm{m}^{-1} \mathrm{~s}^{-1}\right)$

$\mu_{\mathrm{s}} \quad$ Sliding friction coefficient (-)

$\rho \quad$ Time-dependent bulk density during flow propagation $\left(\mathrm{kg} \mathrm{m}^{-3}\right)$

$\rho_{\text {aer }} \quad$ Bulk density after aeration pre-conditioning $\left(\mathrm{kg} \mathrm{m}^{-3}\right)$

$\rho_{\text {air }} \quad$ Air density $\left(\mathrm{kg} \mathrm{m}^{-3}\right)$

$\rho_{\mathrm{p}} \quad$ Bulk density at the random packing state $\left(\mathrm{kg} \mathrm{m}^{-3}\right)$

$\rho_{\mathrm{s}} \quad$ Particle density $\left(\mathrm{kg} \mathrm{m}^{-1} \mathrm{~s}^{-1}\right)$

$\rho_{\text {vac }} \quad$ Bulk density after vacuum pre-conditioning $\left(\mathrm{kg} \mathrm{m}^{-3}\right)$

$\rho_{\text {vib }} \quad$ Bulk density after sample vibration $\left(\mathrm{kg} \mathrm{m}^{-3}\right)$

$\sigma_{z} \quad$ Vertical stress at the base of the poured granular column ( $\left.\mathrm{kPa}\right)$

$\tau \quad$ Nondimensional time $(-)$

$\tau_{\infty} \quad$ Nondimensional ramp ending time of the basal load profile model (-)

$\tau_{0} \quad$ Nondimensional ramp starting time of the basal load profile model (-)

$\tau_{\mathrm{p}} \quad$ Nondimensional central time of the basal load profile peak model (-)

$\tau_{\mathrm{p}}^{\prime} \quad$ Shape coefficient of the basal load profile peak model (-)

$\tau_{\mathrm{r}} \quad$ Nondimensional central time of the basal load profile ramp model (-)

$\tau_{\mathrm{r}}^{\prime} \quad$ Shape coefficient of the basal load profile ramp model (-)

$\phi \quad$ Normalised flow front location (-)

$\boldsymbol{\omega} \quad$ Average angular velocity field $\left(\mathrm{m} \mathrm{s}^{-1}\right)$

\section{Appendix A. Energy balance by DEM simulations}

We validate the energy balance in Eq. (14) with numerical simulations to support our experimental findings. The simulation of granular column collapse tests by the discrete element method (DEM) gives us a direct insight into bulk scale flowability issues by involving the grain scale properties as model parameters, for instance the particle size polydispersity [78. We present new results from our investigations in 74 on the effect of hygroscopicity on the mass mobilisation of granular columns using the open-source software MercuryDPM [79]. 
We implement a linear spring-dashpot particle contact model coupled with a capillary model, assuming the liquid to be distributed onto adsorbed films and capillary bridges between the particles. The published results indicate that liquid contents in the pendular regime lead to the formation of particle aggregates or caking, and thus a decrease in flowability of the granular materials. Therefore, we compare the energy balance of two particle systems of identical monodisperse spheres with initial column aspect ratios $a>2$, but with differing flowability caused by the different liquid contents $w=0.0 \%$ and $7.5 \%$ of the dry and wet granular flows, respectively, as shown in Fig. A.1. The decay of $E_{\text {pot }}$ is attained around $t=0.85 \mathrm{~s}$ after collapse in both cases, reaching final energy decreases of $64 \%$ and $47 \%$ with respect to the initial values. The large mass mobilisation of the dry particle system explains the greater $E_{\text {pot }}$ reduction, as observed in the fertiliser results. The variation of $E_{\text {kin }}$ is governed by the $E_{\text {kin }}^{\text {trans }}$ component-with peak values of $13 \%$ and $5 \%$ of the $E_{\text {tot }}$, respectively for the dry and wet set-ups - whereas the contribution of $E_{\text {kin }}^{\text {rot }}$ is not relevant to the energy balance, consistently with numerical results on non-cohesive granular flows in [80. However, we notice dissimilarities between the $E_{\text {kin }}^{\text {trans }}$ trends of the numerical results compared to the experimental results in terms of their flowability: larger peak $E_{\text {kin }}^{\text {trans }}$ values are found for the oats samples experimentally but for the dry set-up numerically, which shows a more free-flowing behaviour as observed for the fertiliser. This is probably due to different particle shapes of the studied materials, especially that of the oat flakes, which enhances the avalanching behaviour that entails large peak velocities during flow propagation. Overall, the evolution of the energy components in the simulations appears to be analogous to the experimental results for $a>1$ in Fig. 23 thereby validating the energy balance. Moreover, the agreement between the analyses of the simulation results and the near-wall visualisations also supports the plane strain hypothesis on the flow dynamics.

\section{References}

[1] W. E. Engisch, F. J. Muzzio, Method for characterization of loss-in-weight feeder equipment, Powder Technology 228 (2012) 395-403. doi:10.1016/ j.powtec.2012.05.058 


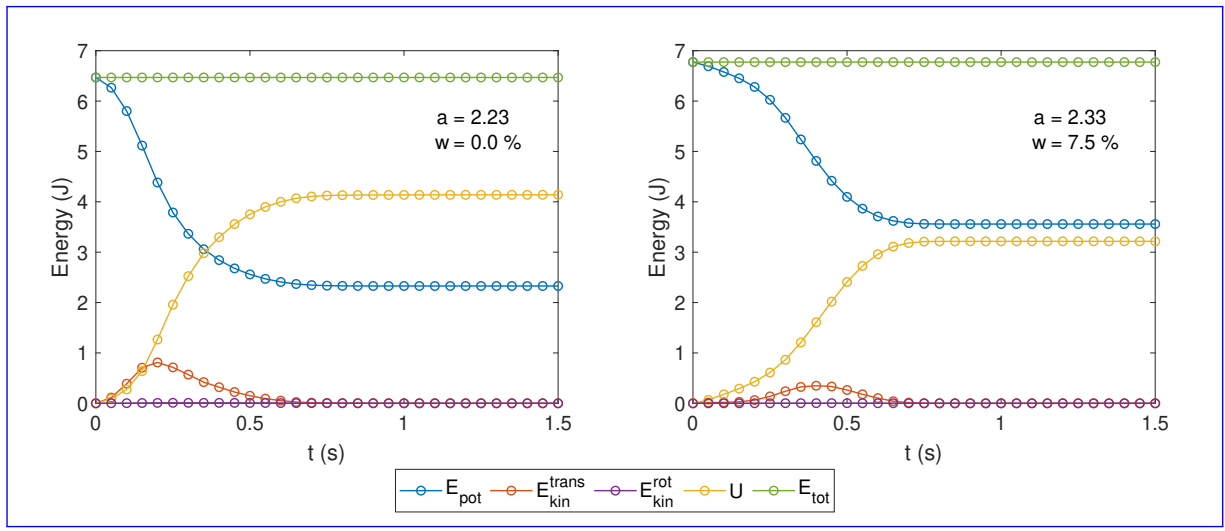

Fig. A.1: Energy balance of dry (left) and wet (right) granular column collapse set-ups used in the DEM simulations.

[2] C. Fahlenbock, Gain-in-weight batching: Choosing system components for maximum accuracy, Powder and Bulk Engineering 17 (9) (2003) 25-32.

[3] D. McGlinchey (Ed.), Bulk Solids Handling: Equipment Selection and Operation, Wiley-Blackwell, 2008.

[4] M. Leturia, M. Benali, S. Lagarde, I. Ronga, K. Saleh, Characterization of flow properties of cohesive powders: A comparative study of traditional and new testing methods, Powder Technology 253 (2014) 406-423. doi: $10.1016 / j$.powtec.2013.11.045.

[5] M. Krantz, H. Zhang, J. Zhu, Characterization of powder flow: Static and dynamic testing, Powder Technology 194 (3) (2009) 239-245. doi: $10.1016 / j$.powtec. 2009.05 .001 .

[6] J. M. N. T. Gray, P. Gajjar, P. Kokelaar, Particle-size segregation in dense granular avalanches, Comptes Rendus Physique 16 (1) (2015) 73-85. doi: $10.1016 / j . c r h y .2015 .01 .004$

[7] M. E. Cates, J. P. Wittmer, J.-P. Bouchaud, P. Claudin, Jamming, Force Chains, and Fragile Matter, Phys. Rev. Lett. 81 (1998) 1841-1844. doi: 10.1103/PhysRevLett.81.1841.

[8] A. J. Matchett, The shape of the cohesive arch in hoppers and silos Some theoretical considerations, Powder Technology 171 (3) (2007) 133 145. doi:10.1016/j.powtec. $2006 \cdot 10.010$ 
[9] K. Johanson, Rathole stability analysis for aerated powder materials, Powder Technology 141 (1) (2004) 161-170. doi:10.1016/j.powtec. 2004.02. 004 .

[10] E. Juarez-Enriquez, G. I. Olivas, P. B. Zamudio-Flores, E. Ortega-Rivas, S. Perez-Vega, D. R. Sepulveda, Effect of water content on the flowability of hygroscopic powders, Journal of Food Engineering 205 (2017) 12-17. doi:10.1016/j.jfoodeng.2017.02.024

[11] O. Pozo, B. Soulestin, N. Olivi-Tran, Stick Slip Motion in Grain Grain Friction in a Humid Atmosphere, Multidiscipline Modeling in Materials and Structures 4 (4) (2008) 393-405. doi:10.1163/157361108785963037.

[12] M. Hartmann, S. Palzer, Caking of amorphous powders - Material aspects, modelling and applications, Powder Technology 206 (1) (2011) 112-121. doi:10.1016/j.powtec. 2010.04 .014

[13] J. J. Fitzpatrick, M. Hodnett, M. Twomey, P. S. M. Cerqueira, J. O'Flynn, Y. H. Roos, Glass transition and the flowability and caking of powders containing amorphous lactose, Powder Technology 178 (2) (2007) 119-128. doi:10.1016/j.powtec. 2007.04 .017

[14] V. Ganesan, K. A. Rosentrater, K. Muthukumarappan, Flowability and handling characteristics of bulk solids and powders - a review with implications for DDGS, Biosystems Engineering 101 (4) (2008) 425-435. doi:10.1016/j.biosystemseng.2008.09.008

[15] J. L. Amorós, G. Mallol, C. Feliu, M. J. Orts, Study of the rheological behaviour of monomodal quartz particle beds under stress. A model for the shear yield functions of powders, Chemical Engineering Science 66 (18) (2011) 4070-4077. doi:10.1016/j.ces.2011.05.034

[16] V. Garg, S. S. Mallick, P. Garcia-Trinanes, R. J. Berry, An investigation into the flowability of fine powders used in pharmaceutical industries, Powder Technology 336 (2018) 375-382. doi:10.1016/j.powtec.2018.06. 014 
[17] M. R. Wu, D. L. Schott, G. Lodewijks, Physical properties of solid biomass, Biomass and Bioenergy 35 (5) (2011) 2093-2105. doi:10.1016/ j.biombioe.2011.02.020

[18] J. Schwedes, Review on testers for measuring flow properties of bulk solids, Granular Matter 5 (1) (2003) 1-43. doi:10.1007/s10035-002-0124-4.

[19] A. B. Spierings, M. Voegtlin, T. Bauer, K. Wegener, Powder flowability characterisation methodology for powder-bed-based metal additive manufacturing, Progress in Additive Manufacturing 1 (1) (2016) 9-20. doi: $10.1007 / \mathrm{s} 40964-015-0001-4$.

[20] U. Zafar, C. Hare, A. Hassanpour, M. Ghadiri, Ball indentation on powder beds for assessing powder flowability: Analysis of operation window, Powder Technology 310 (2017) 300-306. doi:10.1016/j.powtec.2017. 01.047

[21] R. Baserinia, I. C. Sinka, Mass flow rate of fine and cohesive powders under differential air pressure, Powder Technology 334 (2018) 173-182. doi:10.1016/j.powtec.2018.04.041.

[22] G. Lumay, F. Boschini, K. Traina, S. Bontempi, J.-C. Remy, R. Cloots, N. Vandewalle, Measuring the flowing properties of powders and grains, Powder Technology 224 (2012) 19-27. doi:10.1016/j.powtec.2012.02. 015.

[23] G. Lube, H. E. Huppert, R. S. J. Sparks, M. A. Hallworth, Axisymmetric collapses of granular columns, Journal of Fluid Mechanics 508 (2004) 175-199. doi:10.1017/S0022112004009036.

[24] K. A. Holsapple, Modeling granular material flows: The angle of repose, fluidization and the cliff collapse problem, Planetary and Space Science 82-83 (2013) 11-26. doi:10.1016/j.pss.2013.03.001.

[25] G. Lube, H. E. Huppert, R. S. J. Sparks, A. Freundt, Collapses of twodimensional granular columns, Phys. Rev. E 72 (4) (2005) 041301. doi: 10.1103/PhysRevE.72.041301 
[26] E. Lajeunesse, J. B. Monnier, G. M. Homsy, Granular slumping on a horizontal surface, Physics of Fluids 17 (10) (2005) 103302. doi:10.1063/1. 2087687

[27] N. J. Balmforth, R. R. Kerswell, Granular collapse in two dimensions, Journal of Fluid Mechanics 538 (2005) 399-428. doi:10.1017/ S0022112005005537.

[28] S. Siavoshi, A. Kudrolli, Failure of a granular step, Phys. Rev. E 71 (2005) 051302. doi:10.1103/PhysRevE.71.051302.

[29] E. L. Thompson, H. E. Huppert, Granular column collapses: further experimental results, Journal of Fluid Mechanics 575 (2007) 177-186. doi:10.1017/S0022112006004563.

[30] G. Lube, H. E. Huppert, R. S. J. Sparks, A. Freundt, Static and flowing regions in granular collapses down channels, Physics of Fluids 19 (4) (2007) 043301. doi:10.1063/1.2712431.

[31] C. Mériaux, T. Triantafillou, Scaling the final deposits of dry cohesive granular columns after collapse and quasi-static fall, Physics of Fluids 20 (3) (2008) 033301. doi:10.1063/1.2870148.

[32] E. Lajeunesse, A. Mangeney-Castelnau, J. P. Vilotte, Spreading of a granular mass on a horizontal plane, Physics of Fluids 16 (7) (2004) 2371-2381. doi:10.1063/1.1736611.

[33] M. Trepanier, S. V. Franklin, Column collapse of granular rods, Phys. Rev. E 82 (2010) 011308. doi:10.1103/PhysRevE.82.011308

[34] J. M. Warnett, P. Denissenko, P. J. Thomas, M. A. Williams, Collapse of a granular column under rotation, Powder Technology 262 (2014) 249-256. doi:10.1016/j.powtec.2014.04.030

[35] J. M. Warnett, P. Denissenko, P. J. Thomas, E. Kiraci, M. A. Williams, Scalings of axisymmetric granular column collapse, Granular Matter 16 (1) (2014) 115-124. doi:10.1007/s10035-013-0469-x. 
[36] Z. Lai, L. E. Vallejo, W. Zhou, G. Ma, J. M. Espitia, B. Caicedo, X. Chang, Collapse of Granular Columns With Fractal Particle Size Distribution: Implications for Understanding the Role of Small Particles in Granular Flows, Geophysical Research Letters 44 (24) (2017) 12181-12189. doi:10.1002/2017GL075689.

[37] S. K. Bryant, W. A. Take, E. T. Bowman, Observations of grain-scale interactions and simulation of dry granular flows in a large-scale flume, Canadian Geotechnical Journal 52 (5) (2015) 638-655. doi:10.1139/ $\operatorname{cgj}-2013-0425$

[38] A. Mangeney, O. Roche, O. Hungr, N. Mangold, G. Faccanoni, A. Lucas, Erosion and mobility in granular collapse over sloping beds, Journal of Geophysical Research: Earth Surface 115 (2010) F03040. doi:10.1029/ 2009JF001462.

[39] M. Farin, A. Mangeney, O. Roche, Fundamental changes of granular flow dynamics, deposition, and erosion processes at high slope angles: Insights from laboratory experiments, Journal of Geophysical Research: Earth Surface 119 (3) (2014) 504-532. doi:10.1002/2013JF002750.

[40] G. Lube, H. E. Huppert, R. S. J. Sparks, A. Freundt, Granular column collapses down rough, inclined channels, Journal of Fluid Mechanics 675 (2011) 347-368. doi:10.1017/jfm.2011.21.

[41] A. J. Hogg, Two-dimensional granular slumps down slopes, Physics of Fluids 19 (9) (2007) 093301. doi:10.1063/1.2762254.

[42] F. Maeno, A. J. Hogg, R. S. J. Sparks, G. P. Matson, Unconfined slumping of a granular mass on a slope, Physics of Fluids 25 (2) (2013) 023302. doi:10.1063/1.4792707.

[43] G. B. Crosta, S. Imposimato, D. Roddeman, Granular flows on erodible and non erodible inclines, Granular Matter 17 (5) (2015) 667-685. doi: 10.1007/s10035-015-0587-8.

[44] O. Roche, S. Montserrat, Y. Niño, A. Tamburrino, Pore fluid pressure and internal kinematics of gravitational laboratory air-particle flows: Insights 
into the emplacement dynamics of pyroclastic flows, Journal of Geophysical Research: Solid Earth 115 (2010) B09206. doi:10.1029/2009JB007133.

[45] O. Roche, M. Attali, A. Mangeney, A. Lucas, On the run-out distance of geophysical gravitational flows: Insight from fluidized granular collapse experiments, Earth and Planetary Science Letters 311 (3) (2011) 375-385. doi:10.1016/j.epsl.2011.09.023.

[46] R. Artoni, A. C. Santomaso, F. Gabrieli, D. Tono, S. Cola, Collapse of quasi-two-dimensional wet granular columns, Phys. Rev. E 87 (3) (2013) 032205. doi:10.1103/PhysRevE.87.032205.

[47] F. Gabrieli, R. Artoni, A. Santomaso, S. Cola, Discrete particle simulations and experiments on the collapse of wet granular columns, Physics of Fluids 25 (10) (2013) 103303. doi:10.1063/1.4826622.

[48] A. C. Santomaso, S. Volpato, F. Gabrieli, Collapse and runout of granular columns in pendular state, Physics of Fluids 30 (6) (2018) 063301. doi: $10.1063 / 1.5030779$

[49] L. Rondon, O. Pouliquen, P. Aussillous, Granular collapse in a fluid: Role of the initial volume fraction, Physics of Fluids 23 (7) (2011) 073301. doi: $10.1063 / 1.3594200$

[50] A. Bougouin, L. Lacaze, Granular collapse in a fluid: Different flow regimes for an initially dense-packing, Phys. Rev. Fluids 3 (2018) 064305. doi: 10.1103/PhysRevFluids.3.064305.

[51] C.-H. Lee, Z. Huang, M.-L. Yu, Collapse of submerged granular columns in loose packing: Experiment and two-phase flow simulation, Physics of Fluids 30 (12) (2018) 123307. doi:10.1063/1.5050994

[52] J. Torres-Serra, E. Romero, A. Rodríguez-Ferran, J. Caba, X. Arderiu, J.M. Padullés, J. González, Flowability of granular materials with industrial applications - An experimental approach, EPJ Web of Conferences 140 (2017) 03068. doi:10.1051/epjconf/201714003068 
[53] J. Torres-Serra, E. Romero, A. Rodríguez-Ferran, A New Granular Column Collapse Device to Characterise Flowability of Bulk Materials, Proceedings 2 (8) (2018) 488. doi:10.3390/ICEM18-05389

[54] Y. Forterre, O. Pouliquen, Flows of Dense Granular Media, Annual Review of Fluid Mechanics 40 (1) (2008) 1-24. doi:10.1146/annurev.fluid.40. 111406.102142

[55] R. T. Schneider, Pneumatics and vacuum perform in packaging equipment, Hydraulics \& Pneumatics 50 (9) (1997) 45-46.

[56] D. Gollin, W. Brevis, E. T. Bowman, P. Shepley, Performance of PIV and PTV for granular flow measurements, Granular Matter 19 (3) (2017) 42. doi:10.1007/s10035-017-0730-9.

[57] A. J. Holyoake, J. N. McElwaine, High-speed granular chute flows, Journal of Fluid Mechanics 710 (2012) 35-71. doi:10.1017/jfm.2012.331

[58] L. Sarno, A. Carravetta, Y.-C. Tai, R. Martino, M. Papa, C.-Y. Kuo, Measuring the velocity fields of granular flows - Employment of a multi-pass two-dimensional particle image velocimetry (2D-PIV) approach, Advanced Powder Technology 29 (12) (2018) 3107-3123. doi:10.1016/j.apt.2018. 08.014

[59] B. Turnbull, E. T. Bowman, J. N. McElwaine, Debris flows: Experiments and modelling, Comptes Rendus Physique 16 (1) (2015) 86-96. doi:10. 1016/j.crhy.2014.11.006.

[60] N. Sanvitale, E. T. Bowman, Visualization of dominant stress-transfer mechanisms in experimental debris flows of different particle-size distribution, Canadian Geotechnical Journal 54 (2) (2017) 258-269. doi: 10.1139/cgj-2015-0532.

[61] T. Xu, Y.-C. Jin, Y.-C. Tai, C.-H. Lu, Simulation of velocity and shear stress distributions in granular column collapses by a mesh-free method, Journal of Non-Newtonian Fluid Mechanics 247 (2017) 146-164. doi:10. $1016 / j \cdot j n n f m .2017 .07 .003$ 
[62] S. J. de Vet, B. Yohannes, K. M. Hill, J. R. de Bruyn, Collapse of a rectangular well in a quasi-two-dimensional granular bed, Phys. Rev. E 82 (2010) 041304. doi:10.1103/PhysRevE.82.041304.

[63] W. Thielicke, E. J. Stamhuis, PIVlab - Towards User-friendly, Affordable and Accurate Digital Particle Image Velocimetry in MATLAB, Journal of Open Research Software 2 (1) (2014) e30. doi:10.5334/jors.b1.

[64] A. Masullo, R. Theunissen, Automated mask generation for PIV image analysis based on pixel intensity statistics, Experiments in Fluids 58 (6) (2017) 70. doi:10.1007/s00348-017-2357-3.

[65] J. M. Boac, M. E. Casada, R. G. Maghirang, J. P. Harner III, Material and Interaction Properties of Selected Grains and Oilseeds for Modeling Discrete Particles, Transactions of the ASABE 53 (4) (2010) 1201-1216. doi:10.13031/2013.32577.

[66] T. Oishi, M. Goto, A. Kasahara, M. Tosa, Low frictional copper oxide film prepared with sodium hydroxide solution, Surface and Interface Analysis 36 (8) (2004) 1259-1261. doi:10.1002/sia.1889

[67] X. Chen, A. S. Elwood Madden, Z. Reches, The frictional strength of talc gouge in high-velocity shear experiments, Journal of Geophysical Research: Solid Earth 122 (5) (2017) 3661-3676. doi:10.1002/2016JB013676.

[68] S. J. Blott, K. Pye, Particle shape: a review and new methods of characterization and classification, Sedimentology 55 (1) (2008) 31-63. doi: $10.1111 / j .1365-3091.2007 .00892 . x$

[69] T. H. Druitt, G. Avard, G. Bruni, P. Lettieri, F. Maez, Gas retention in fine-grained pyroclastic flow materials at high temperatures, Bulletin of Volcanology 69 (8) (2007) 881-901. doi:10.1007/s00445-007-0116-7.

[70] J. R. van Ommen, J. M. Valverde, R. Pfeffer, Fluidization of nanopowders: a review, Journal of Nanoparticle Research 14 (3) (2012) 737. doi:10. 1007/s11051-012-0737-4.

[71] D. Geldart, E. C. Abdullah, A. Hassanpour, L. C. Nwoke, I. Wouters, Characterization of powder flowability using measurement of angle of repose, 
China Particuology 4 (3) (2006) 104-107. doi:10.1016/S1672-2515(07) $60247-4$

[72] E. J. Fern, K. Soga, The role of constitutive models in MPM simulations of granular column collapses, Acta Geotechnica 11 (3) (2016) 659-678. doi:10.1007/s11440-016-0436-x.

[73] Y. Guo, J. S. Curtis, Discrete Element Method Simulations for Complex Granular Flows, Annual Review of Fluid Mechanics 47 (1) (2015) 21-46. doi:10.1146/annurev-fluid-010814-014644.

[74] J. Torres-Serra, E. Romero, A. Rodríguez-Ferran, Hygroscopicity issues in powder and grain technology, in: C. W. W. Ng, A. K. Leung, A. C. F. Chiu, C. Zhou (Eds.), 7th International Conference on Unsaturated Soils, UNSAT 2018, Hong Kong University of Science and Technology, 2018, pp. 805-810.

[75] J. Torres-Serra, J. Caba, A. Rodríguez-Ferran, E. Romero, Classifying granular materials for feeding performance using experimental and numerical techniques, in: 9th International Conference on Conveying and Handling of Particulate Solids, CHoPS 2018, 2018.

[76] A. Podlozhnyuk, S. Pirker, C. Kloss, Efficient implementation of superquadric particles in Discrete Element Method within an open-source framework, Computational Particle Mechanics 4 (1) (2017) 101-118. doi: 10.1007/s40571-016-0131-6.

[77] D. R. Tunuguntla, A. R. Thornton, T. Weinhart, From discrete elements to continuum fields: Extension to bidisperse systems, Computational Particle Mechanics 3 (3) (2016) 349-365. doi:10.1007/s40571-015-0087-y.

[78] J. Torres-Serra, D. R. Tunuguntla, I. F. C. Denissen, A. Rodríguez-Ferran, E. Romero, Discrete element modelling of granular column collapse tests with industrial applications, in: P. Wriggers, M. Bischoff, E. Oñate, D. Owen, T. Zohdi (Eds.), 5th International Conference on Particle-Based Methods - Fundamentals and Applications, PARTICLES 2017, International Center for Numerical Methods in Engineering, 2017, pp. 530-538. 
[79] T. Weinhart, D. R. Tunuguntla, M. P. v. S. Lantman, I. F. Denissen, C. R. Windows-Yule, H. Polman, J. M. Tsang, B. Jin, L. Orefice, K. van der Vaart, S. Roy, H. Shi, A. Pagano, W. den Breeijen, B. J. Scheper, A. Jarray, S. Luding, A. R. Thornton, MercuryDPM: Fast, flexible particle simulations in complex geometries part II: Applications, in: P. Wriggers, M. Bischoff, E. Oñate, D. Owen, T. Zohdi (Eds.), 5th International Conference on Particle-Based Methods - Fundamentals and Applications, PARTICLES 2017, International Center for Numerical Methods in Engineering, 2017, pp. $123-134$.

[80] S. Utili, T. Zhao, G. T. Houlsby, 3D DEM investigation of granular column collapse: Evaluation of debris motion and its destructive power, Engineering Geology 186 (2015) 3-16. doi:10.1016/j.enggeo.2014.08.018. 